\title{
Luminescence Imaging of Acute Liver Injury by Biodegradable and
}

\section{Biocompatible Nanoprobes}

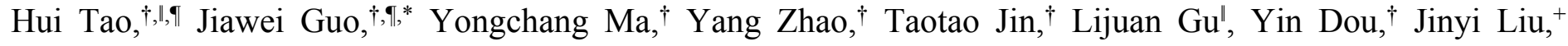
Houyuan $\mathrm{Hu}, \stackrel{\ddagger}{ }$ Xiaoxing Xiong,,$^{\prime, *}$ and Jianxiang Zhang ${ }^{*}, \dagger, \perp$

†Department of Pharmaceutics, College of Pharmacy, Third Military Medical University (Army Medical University), Chongqing 400038, China

"Central Laboratory, Renmin Hospital of Wuhan University, Wuhan 430006, China

${ }^{+}$Institute of Toxicology, College of Preventive Medicine, Third Military Medical University (Army Medical University), Chongqing 400038, China

†. Chongqing 400038, China

${ }^{\perp}$ State Key Laboratory of Trauma, Burn and Combined Injury, Institute of Combined Injury, Third Military Medical University (Army Medical University), Chongqing 400038, China

\section{Corresponding authors:}

Jianxiang Zhang, PhD, Prof.

E-mail: jxzhang1980@gmail.com,jxzhang@tmmu.edu.cn

Xiaoxing Xiong, PhD, Prof.

E-mail: xiaoxingxiong@whu.edu.cn

Jiawei Guo, PhD, Associate Prof.

E-mail: breakhp@163.com 


\section{Supplementary methods}

Materials. Luminol, luminol sodium salt, $\alpha$-cyclodextrin ( $\alpha$-CD), 1,1'-carbonyldiimidazole (CDI), anhydrous dimethylformamide (DMF), 4-aminobenzoic acid hydrazide (4-ABAH), Tempol, phorbol 12-myristate 13-acetate (PMA), thioglycollate, lipopolysaccharides (LPS, O26: B6), Oil Red O (ORO), 2',7'-dichlorofluorescin diacetate (DCFH-DA), and 4',6-diamidino-2-phenylindole (DAPI) were purchased from Sigma-Aldrich (USA). Myeloperoxidase (MPO) was obtained from BioVision, Inc. (California, USA). Fetal bovine serum (FBS) was obtained from Gibco (USA). Anti-mouse Ly6G antibody and mouse MPO antibody were purchased from BioLegend, Co. (San Diego, USA) and R\&D Systems Inc. (Minneapolis, USA), respectively. 1,2-Distearoyl-sn-glycero-3-phosphoethanolamine- $N$-[maleimide-2000] (DSPE-PEG-Maleimide) was purchased from Nanosoft Polymers (Winston-Salem NC, USA). N-Acetyl Pro-Gly-Pro-Cys (PGP) peptide was obtained from Sangon Biotech, Co. Ltd (Shanghai, China). Cyanine 5 NHS ester (Cy5) and Cyanine 7.5 NHS ester (Cy7.5) was obtained from Lumiprobe, Co. (Hallandale Beach, USA). ELISA kits were purchased from Boster Biological Technology Co. Ltd (USA). Amplex Red Hydrogen Peroxide/Peroxidase Assay Kit was obtained from Thermo Fisher Scientific (USA). Carbon tetrachloride $\left(\mathrm{CCl}_{4}\right)$ and olive oil was purchased from Aladdin (Shanghai, China). Anti-mouse V450-CD11b antibody, anti-mouse PE-Ly6G antibody, and anti-mouse FITC-F4/80 antibody were purchased from BD Biosciences (USA).

Materials Characterization. Fourier-transform infrared (FT-IR) spectra were recorded on a PerkinElmer FT-IR spectrometer (100S, USA). UV-Vis spectroscopy was conducted on an ultraviolet spectrophotometer (TU-1901, Beijing Purkinje General instrument, China). ${ }^{1} \mathrm{H}$ NMR and ${ }^{13} \mathrm{C}$ NMR spectra were acquired on a nuclear magnetic resonance (NMR) spectrometer operating at $600 \mathrm{MHz}$ (DD2, Agilent). Matrix-assisted laser desorption/ionization time-of-flight (MALDI-TOF) mass spectrometry was carried out on a Micromass TofSpec 2E spectrometer (Waters).

Characterization of NPs. Particle size, size distribution profiles, and $\zeta$-potential values of NPs were detected with a Malvern Zetasizer Nano ZS instrument at $25^{\circ} \mathrm{C}$. Transmission electron microscopy (TEM) 
observation was conducted on a TECNAI-10 microscope (Philips, Netherlands). Scanning electron microscopy (SEM) was performed with a FIB-SEM microscope (Crossbeam 340, Zeiss).

In Vitro Stability and Hydrolysis of LaCD NPs. For in vitro stability tests, $4 \mathrm{mg}$ freshly produced LaCD $\mathrm{NP}$ was dispersed in $2 \mathrm{~mL}$ of deionized water at 25 or $37^{\circ} \mathrm{C}$. After $48 \mathrm{~h}$, particle size and $\zeta$-potential values of LaCD NP were detected. For hydrolysis tests, $2 \mathrm{mg}$ LaCD NP was dispersed in $2 \mathrm{~mL}$ of PBS (0.01 M, pH 7.4) containing various concentrations of $\mathrm{H}_{2} \mathrm{O}_{2}$ or $\mathrm{NaClO}$ at $37^{\circ} \mathrm{C}$. At predetermined time points, the transmittance values of NP-containing aqueous solutions were measured at $500 \mathrm{~nm}$. The degree of hydrolysis was calculated based on the transmittance values. Similar procedures were used to examine hydrolysis profiles of LaCD NP in solutions with varied $\mathrm{pH}$ values.

Cytotoxicity Evaluation of LaCD NP in Different Cell Lines. RAW264.7 murine macrophages, human umbilical vein endothelial cells (HUVECs), and HepG2 human liver cancer cells were seeded in 96-well plates at a density of $1 \times 10^{4}$ per well. Cells were incubated under standard conditions overnight, and then they were treated with LaCD NP at different doses for another $12 \mathrm{~h}$. The cell viability was quantified by MTT assay.

Isolation of Mouse Peritoneal Neutrophils. To induce the production of neutrophils, BALB/c mice were intraperitoneally (i.p.) injected with $3 \mathrm{wt} \%$ thioglycolate. After $4 \mathrm{~h}$, peritoneal cells were collected with cold HBSS. Cells were incubated with DMEM containing 10\% FBS, penicillin, and streptomycin. After 10 min, non-adherent cells were removed by gentle washing with HBSS. Then peritoneal neutrophils were collected and stained with PE-Ly6G and V450-CD11b antibodies, the purity of neutrophils was confirmed by flow cytometry.

Hydrolysis of LaCD NP in Neutrophils and Liver Homogenates. Peritoneal neutrophils $\left(1.2 \times 10^{7 / \text { well })}\right.$ were planted in 12-well plates, which were first stimulated with $200 \mathrm{ng}$ PMA. After $1 \mathrm{~h}, 20 \mathrm{mg}$ freshly produced LaCD NP was added. After hydrolysis for 7 days, the samples were lyophilized. On the other hand, liver tissues of $\mathrm{C} 57 \mathrm{BL} / 6$ mice were isolated at $24 \mathrm{~h}$ after administration of $\mathrm{CCl}_{4}$, which were minceded and incubated in HBSS with $0.05 \%$ collagenase IV for $1 \mathrm{~h}$ at $37^{\circ} \mathrm{C}$. The digested tissues were passed through a $70-\mu \mathrm{m}$ nylon cell strainer to remove undigested tissues and the cell suspension was reserved. Then $20 \mathrm{mg}$ freshly produced LaCD NP was added. After incubation for 7 days, the samples were lyophilized. In both cases, 
the hydrolyzed products were characterized by ${ }^{1} \mathrm{H}$ NMR spectroscopy and MALDI-TOF spectrometry.

Fluorescence Microscopy Observation of ROS in Neutrophils. Peritoneal neutrophils isolated from BALB/c mice were first stimulated with $100 \mathrm{ng}$ PMA. Cells treated with fresh medium served as the control. After $1 \mathrm{~h}, 50 \mu \mathrm{M}$ DCFH-DA was added and cells were incubated for another $30 \mathrm{~min}$. Cells were washed with PBS, fixed with 4\% paraformaldehyde, and stained with DAPI, followed by CLSM observation.

Quantification of MPO in Activated Neutrophils. Peritoneal neutrophils were seeded in a 12-well culture plate at a density of $5 \times 10^{5}$ per well. After $10 \mathrm{~min}$, non-adherent cells were removed by gentle washing with HBSS and neutrophils were treated with or without $100 \mathrm{ng}$ PMA for $1 \mathrm{~h}$. Then, cells were digested and MPO in the supernatant was assessed by ELISA assay.

Quantification of Different Mediators in Liver Tissues of ALI Mice. At defined time points post initiation of ALI in C57BL/6 mice, liver tissues were harvested, which were minceded and incubated in HBSS with $0.05 \%$ collagenase IV for $1 \mathrm{~h}$ at $37^{\circ} \mathrm{C}$. Then the digested tissues were passed through a $70-\mu \mathrm{m}$ nylon cell strainer, and the cell suspension was centrifuged at $50 \mathrm{~g}$ for $3 \mathrm{~min}$ to remove hepatocytes and large debris. The supernatant was centrifuged at $450 \mathrm{~g}$ for $10 \mathrm{~min}$, followed by incubation with ACK lysis buffer for 5 min. Subsequently, $150 \mu \mathrm{L}$ of Triton X-100 (0.5 wt\%) was added into $1 \mathrm{~mL}$ of the collected cell suspension, which was centrifuged at $16000 \mathrm{~g}$ for $10 \mathrm{~min}$ at $4^{\circ} \mathrm{C}$. The levels of tumor necrosis factor- $\alpha$ (TNF- $\alpha$ ), interleukin- $1 \beta$ (IL-1 $\beta$ ), MPO, and $\mathrm{H}_{2} \mathrm{O}_{2}$ in the supernatant were assessed by ELISA assay.

\section{Quantification of Neutrophils, Different Mediators, Biochemical Markers, and Histological Analyses} for ALF Mice. At defined time points post exposure to $\mathrm{CCl}_{4}$ by i.p. injection in $\mathrm{C} 57 \mathrm{BL} / 6$ mice, neutrophils in liver tissues were quantified by flow cytometry. The levels of TNF- $\alpha$, IL-1 $\beta$, MPO, and $\mathrm{H}_{2} \mathrm{O}_{2}$ in liver tissues were determined by ELISA assay. In addition, blood samples were collected for quantification of serum levels of ALT and AST. Histological sections of hepatic tissues were also prepared and stained by H\&E.

Also, the neutrophil count and MPO level in the livers were analyzed by immunofluorescence. To this end, histological sections were separately incubated with antibodies to Cy3-labeled Ly6G and FITC-labeled MPO. After nuclei were counterstained with DAPI, the cryosections were imaged by confocal microscopy. 
Isolation and Identification of Kupffer Cells. Briefly, after C57BL/6 mice were anaesthetized, the portal vein was inserted with a plastic catheter and the liver was perfused in situ with $10 \mathrm{~mL}$ of HBSS to drive out red blood cells. After perfusion with another $10 \mathrm{~mL}$ of $0.1 \%$ collagenase IV solution, livers were excised, minceded, and transferred to a 6 -well plate. Subsequently, the liver homogenates were incubated with $0.1 \%$ collagenase IV at $37^{\circ} \mathrm{C}$ for $30 \mathrm{~min}$. Following digestion, the liver homogenates were passed through a $70-\mu \mathrm{m}$ nylon cell strainer to remove undigested tissues. Then the cell suspension was centrifuged at $450 \mathrm{~g}$ for $3 \mathrm{~min}$ at $4^{\circ} \mathrm{C}$, and the cell sediments were retained and resuspended with RPMI-1640 medium, followed by centrifugation at $50 \mathrm{~g}$ for $3 \mathrm{~min}$ at $4{ }^{\circ} \mathrm{C}$. Subsequently, the supernatant was centrifuged at $450 \mathrm{~g}$ for $3 \mathrm{~min}$, and the cell sediments were resuspended with RPMI-1640 medium. To indentify Kupffer cells, cells were incubated with fluorescent-labeled CD11b and F4/80 antibodies for $30 \mathrm{~min}$ and then analyzed by flow cytometry.

Quantification of Kupffer Cells in Livers of Mice with ALF. To induce ALF in C57BL/6 mice, animals were administered $\mathrm{CCl}_{4}$ by i.p. injection. The Kupffer cells in the livers of ALF or healthy mice were isolated according to the above mentioned procedures. Then the obtained cell suspensions were stained with CD11b and F4/80 antibodies for analysis by flow cytometry.

The Effects of Macrophages on Cellular Uptake or Luminescence of LaCD-Derived NPs in Neutrophils. Peritoneal macrophages were used as a substitute for Kupffer cells. Both peritoneal neutrophils and macrophages were isolated from BALB/c mice induced with $3 \mathrm{wt} \%$ thioglycollate. Then $4 \times 10^{5}$ neutrophils and $3 \times 10^{5}$ macrophages were planted in each well of 12-well plates. In the control group, only peritoneal neutrophils $\left(4 \times 10^{5}\right)$ were planted. After $10 \mathrm{~min}, \mathrm{Cy} 5 / \mathrm{LaCD}-\mathrm{PEG}$ NP or Cy5/LaCD-PEG-PGP NP at $50 \mu \mathrm{g} / \mathrm{mL}$ was added and incubated for $1 \mathrm{~h}$. Fluorescence intensities were quantified by flow cytometry.

To examine the effect of macrophages on luminescence of LaCD-based NPs in neutrophils, peritoneal neutrophils were cocultured with macrophages in 12-well plates. After stimulation with 100 ng PMA, cells in each group were treated with $3 \mathrm{mg} / \mathrm{mL}$ LaCD-PEG NP or LaCD-PEG-PGP NP. The luminescence signals in different groups were examined by IVIS imaging system with an exposure time of $5 \mathrm{~min}(\mathrm{f} / \mathrm{stop}=1$, binning $=$ 8, no optical filter). 
Hemolysis Tests of LaCD NP. Blood samples were collected from male Sprague-Dawley rats, from which $2 \%$ erythrocyte suspension in saline was prepared. The suspension of erythrocytes was separately mixed with LaCD NP at different concentrations, with a total volume of $1 \mathrm{~mL}$. The final concentration of LaCD NP varied from $0.05,0.2,0.6$, to $1.0 \mathrm{mg} / \mathrm{mL}$. In the positive control group, deionized water was added, while saline solution was added in the negative control group. The mixed suspensions were incubated at $37^{\circ} \mathrm{C}$. After $2 \mathrm{~h}$, all samples were centrifuged at $200 \mathrm{~g}$ for $5 \mathrm{~min}$. The optical density of supernatant was measured at $570 \mathrm{~nm}$, and the degree of hemolysis was calculated.

Acute Toxicity Tests. BALB/c mice were randomly divided into 3 groups $(n=6)$. Mice in the LaCD NP group were i.v. administered with $\mathrm{LaCD} \mathrm{NP}$ at 500 or $1000 \mathrm{mg} / \mathrm{kg}$, while mice in the control group were i.v. injected with saline. After administration, the body weight of mice and their general behaviors were monitored for any signs of disease each day. At day 15, mice were euthanized. Blood samples were collected for hematological analysis and quantification of biochemical markers relevant to liver/kidney functions. Major organs including heart, liver, spleen, lung, and kidney were harvested and weighed. Histological sections were prepared and stained with H\&E. 


\section{Supplementary results}

A
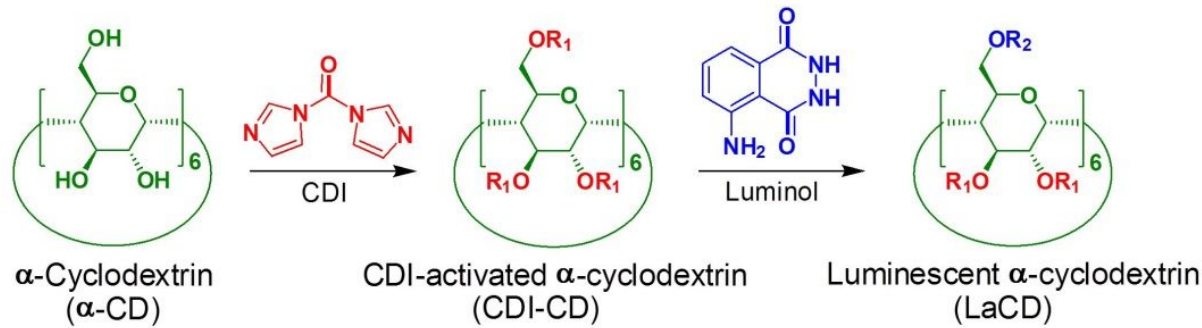

$\underset{(\alpha-C D)}{\alpha-C y c l o d e x t r i n}$

CDI-activated $\alpha$-cyclodextrin (CDI-CD)

Luminescent $\alpha$-cyclodextrin
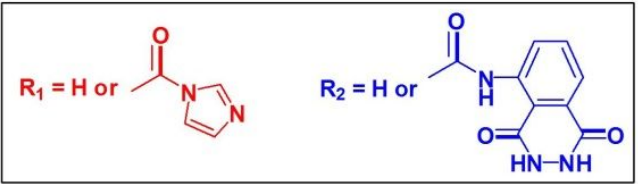

B
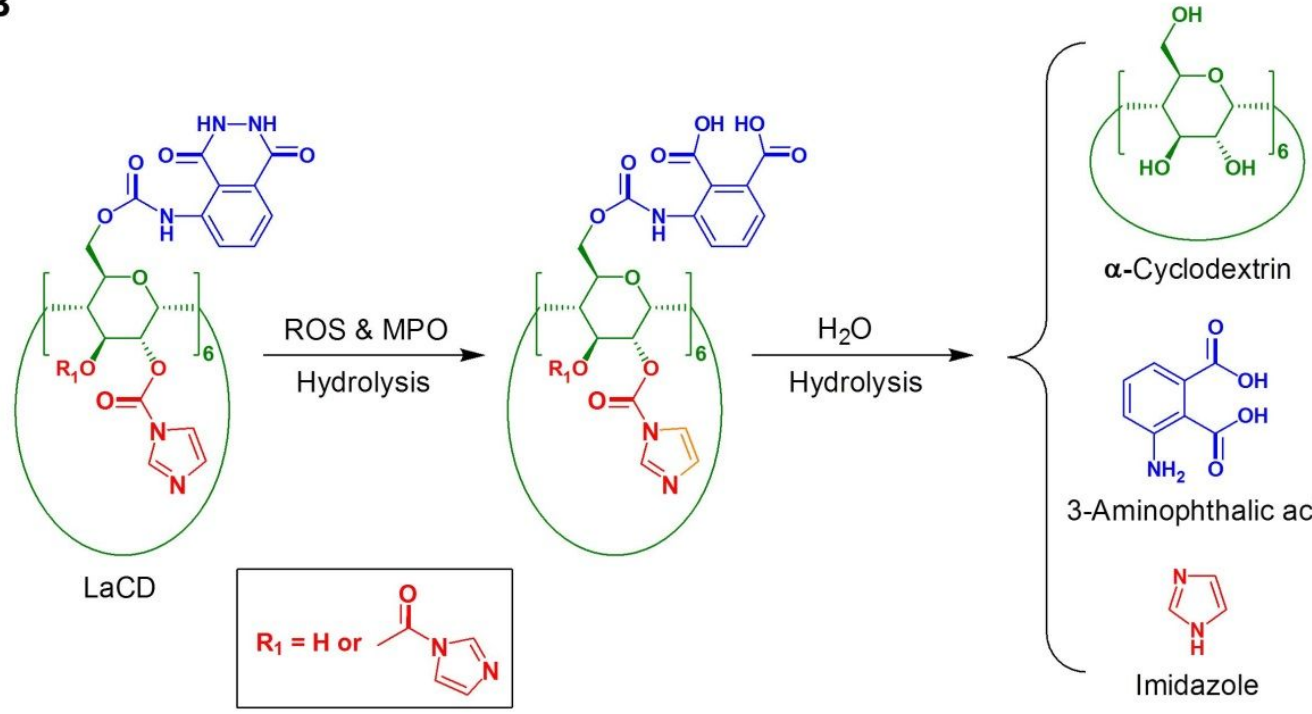

3-Aminophthalic acid

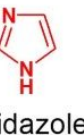

Figure S1. Schematic illustration of synthesis and hydrolysis of a luminescent material of luminol-conjugated $\alpha$-CD (LaCD). (A) The synthetic route of $\mathrm{LaCD}$ by functional modification of $\alpha$-cyclodextrin $(\alpha-\mathrm{CD})$. (B) Hydrolysis of $\mathrm{LaCD}$ into the parent $\alpha-\mathrm{CD}$ compound and water-soluble products in the presence of ROS and MPO. 
A

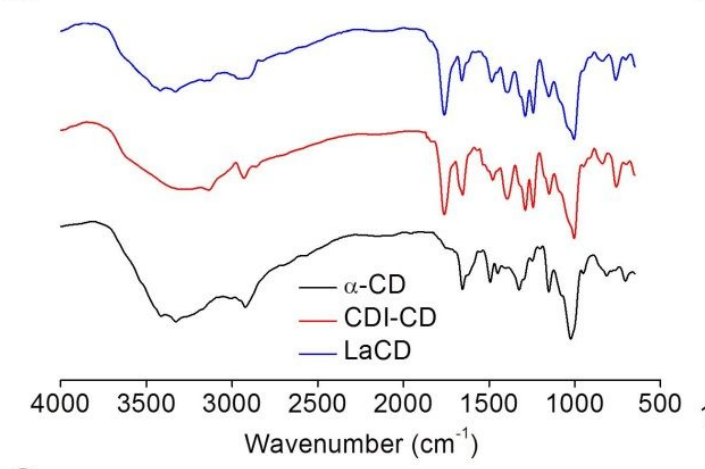

C

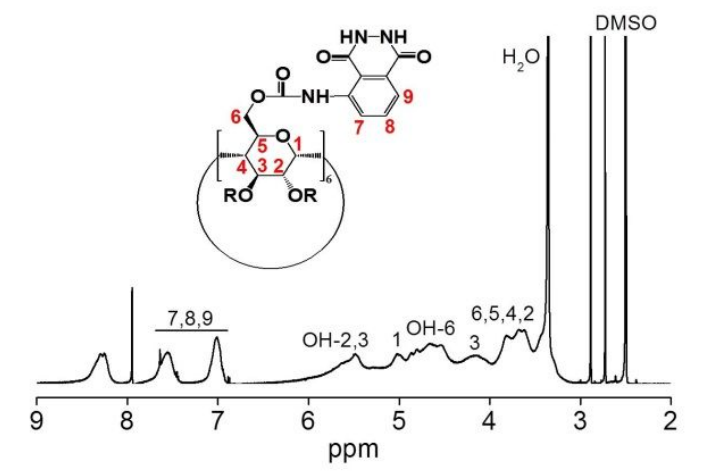

B

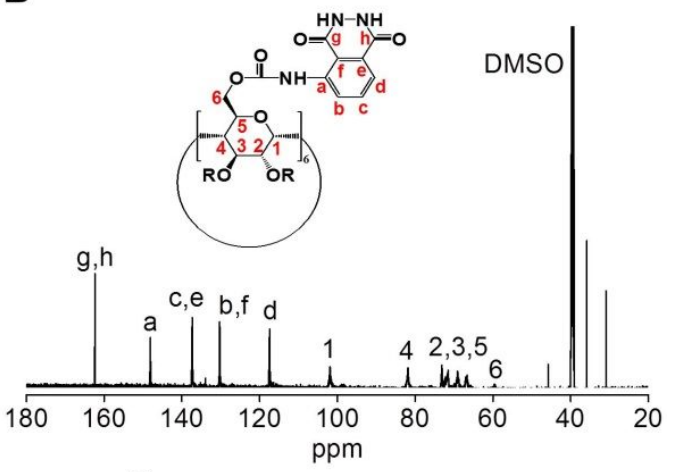

D

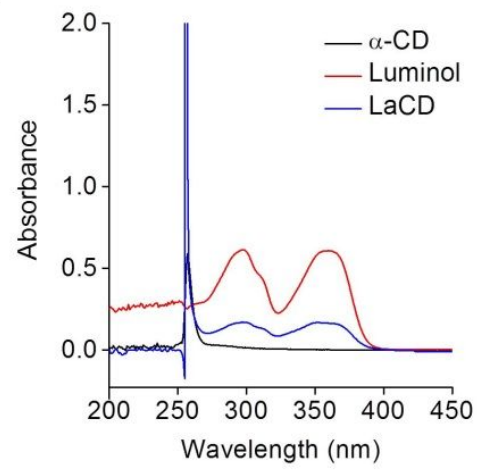

E

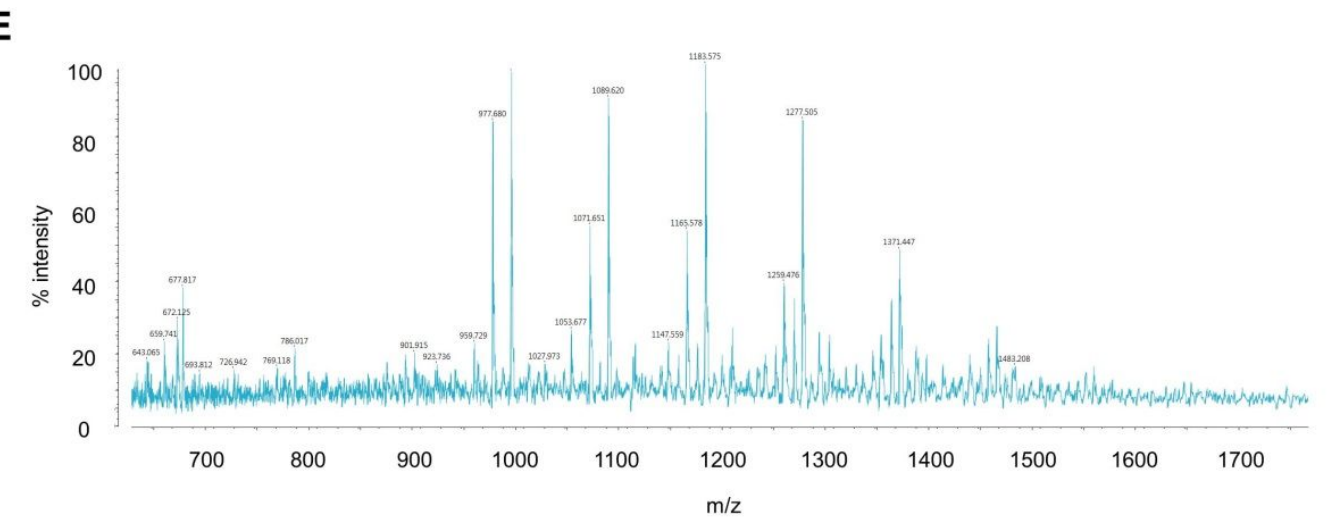

Figure S2. Characterization of a luminescent material based on luminol-conjugated $\alpha$-CD (LaCD). (A) FT-IR spectra of different materials including $\alpha-\mathrm{CD}$, CDI-activated $\alpha-\mathrm{CD}$ (CDI-CD), and luminol-conjugated $\alpha-\mathrm{CD}$ (LaCD). (B,C) ${ }^{13} \mathrm{C}$ NMR (B) and ${ }^{1} \mathrm{H}$ NMR (C) spectra of LaCD in DMSO-d $\mathrm{d}_{6}$. (D) UV-Vis absorbance spectra of different materials in DMSO. (E) Matrix-assisted laser desorption/ionization time-of-flight (MALDI-TOF) mass spectrum of LaCD. 


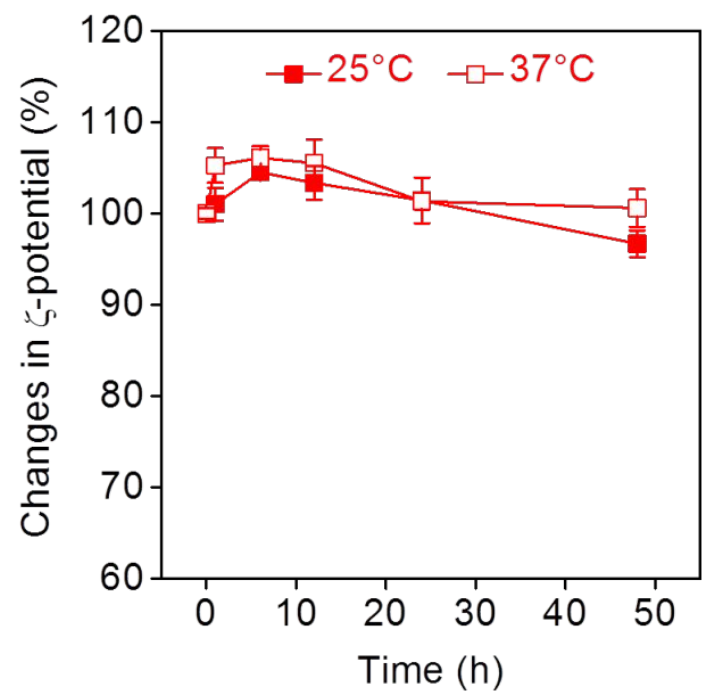

Figure S3. Changes in relative $\zeta$-potential values of $\mathrm{LaCD}$ NP in deionized water during $48 \mathrm{~h}$ of incubation at 25 or $37^{\circ} \mathrm{C}$. Data are presented as means $\pm \mathrm{SD}(\mathrm{n}=4)$. 
A

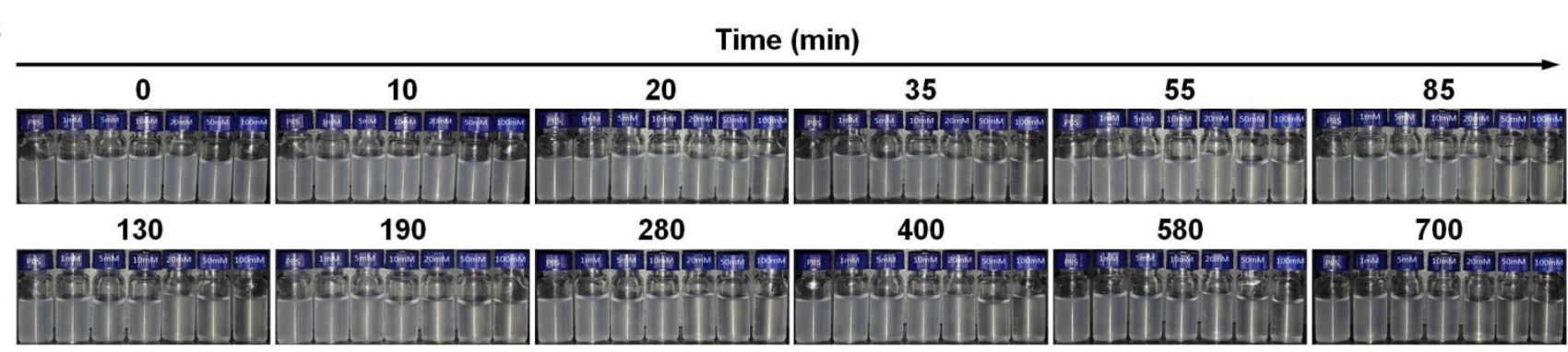

B

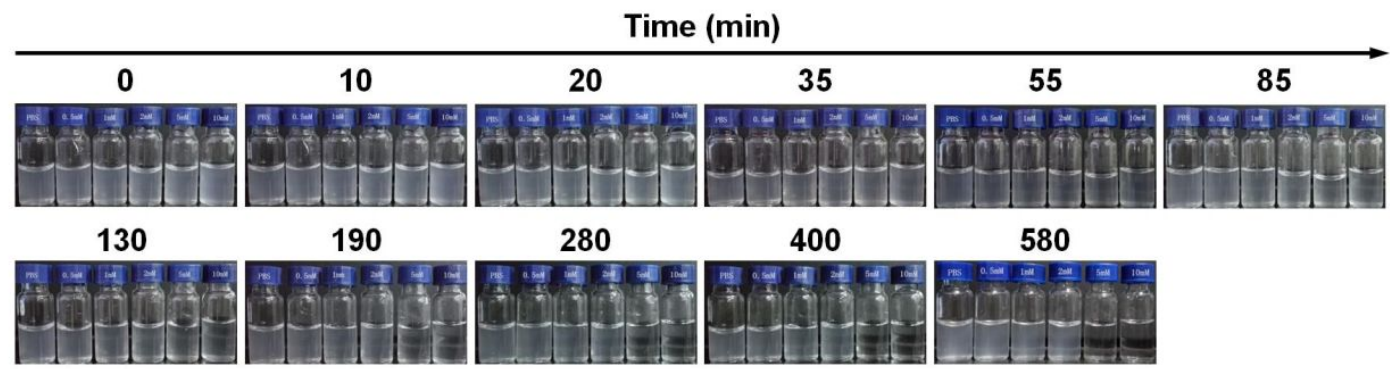

C

Time (min)

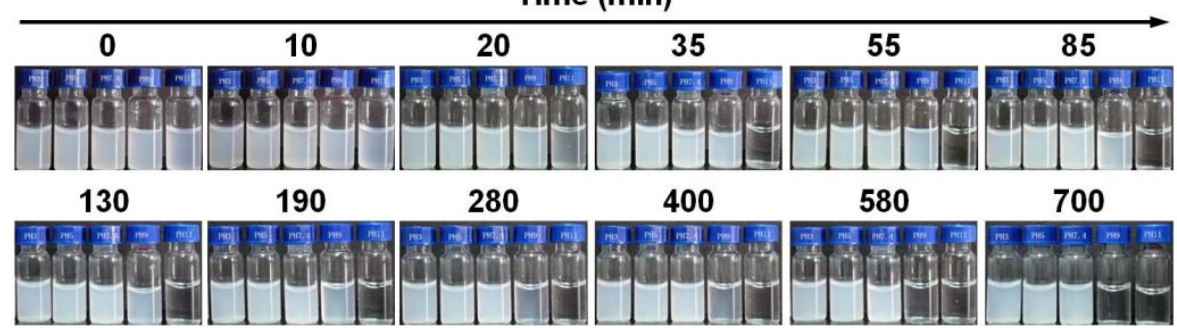

Figure S4. Digital photos showing hydrolysis of LaCD NP. (A-C) Hydrolysis profiles of LaCD NP in the presence of various concentrations of $\mathrm{H}_{2} \mathrm{O}_{2}$ (A), $\mathrm{NaClO}$ (B), or at different $\mathrm{pH}$ values (C). For $\mathrm{H}_{2} \mathrm{O}_{2}$, its concentration varied from $0,1,5,10,20,50$, to $100 \mathrm{mM}$ (from left to right). As for $\mathrm{NaClO}$, its concentration varied from $0,0.5,1,2,5$, to $10 \mathrm{mM}$ (from left to right). In the case of the $\mathrm{pH}$ value, it varied from 3, 5, 7.4, 9, to 11 (from left to right). 
A

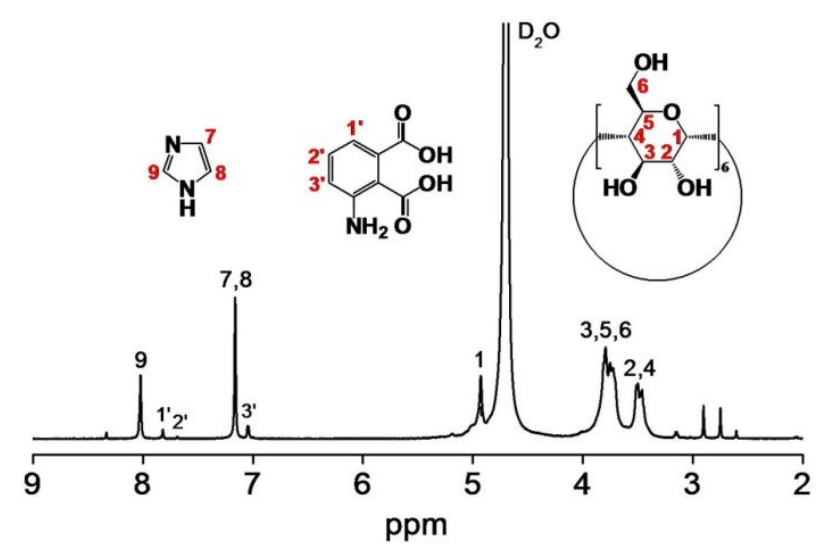

B

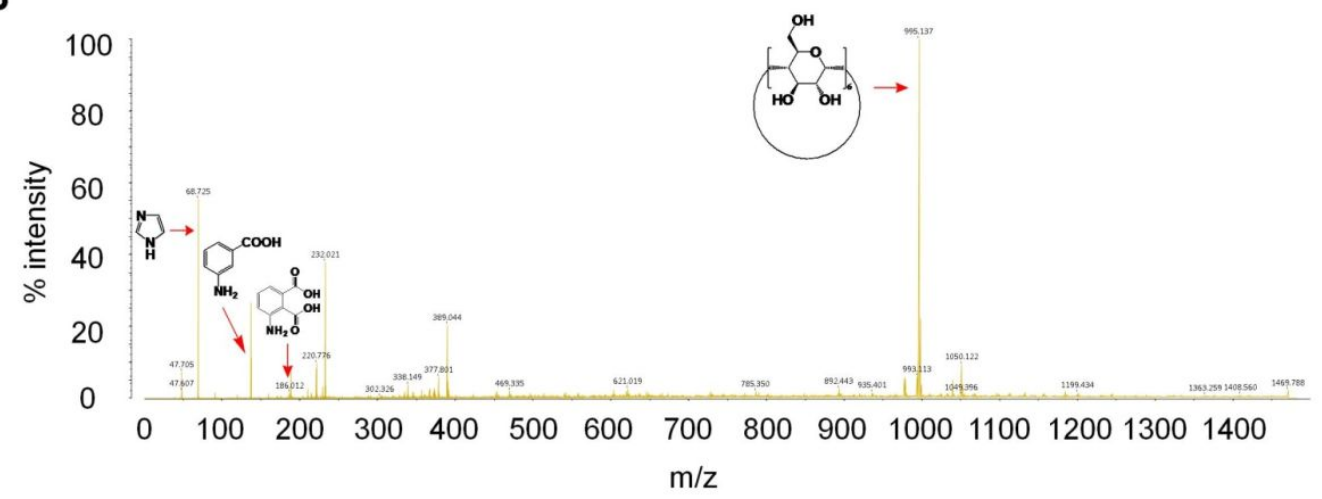

Figure S5. Characterization of the hydrolyzed products of LaCD. (A) A typical ${ }^{1} \mathrm{H}$ NMR spectrum of the hydrolyzed sample of LaCD in $\mathrm{D}_{2} \mathrm{O}$. Hydrolysis of LaCD was performed in the presence of $1 \mathrm{M} \mathrm{H}_{2} \mathrm{O}_{2}$, and then the sample was lyophilized for ${ }^{1} \mathrm{H}$ NMR measurement. (B) Mass spectrum of hydrolyzed LaCD. LaCD was hydrolyzed in $1 \mathrm{M} \mathrm{H}_{2} \mathrm{O}_{2}$, followed by lyophilization. 
A

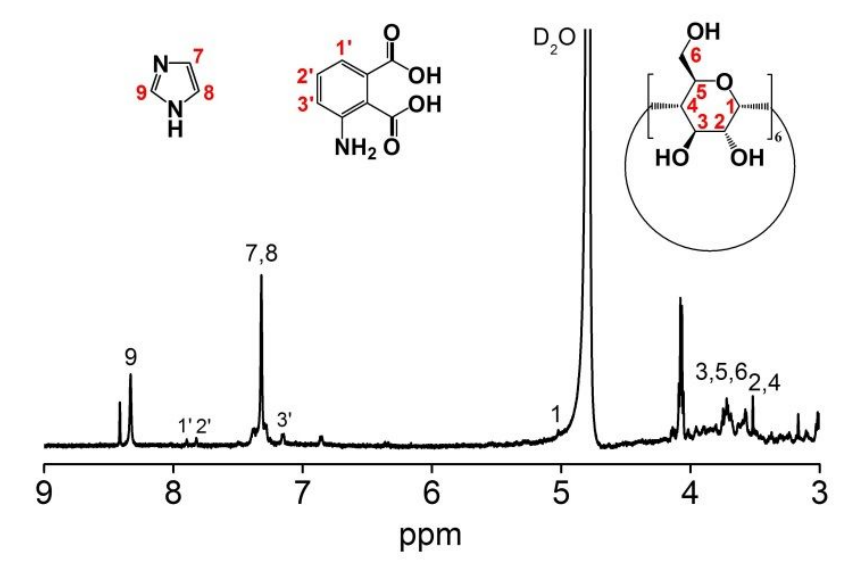

C

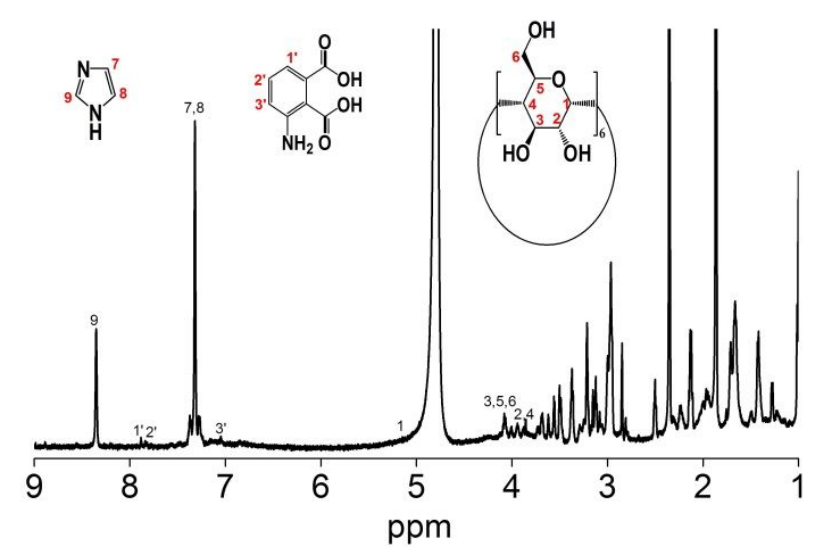

B

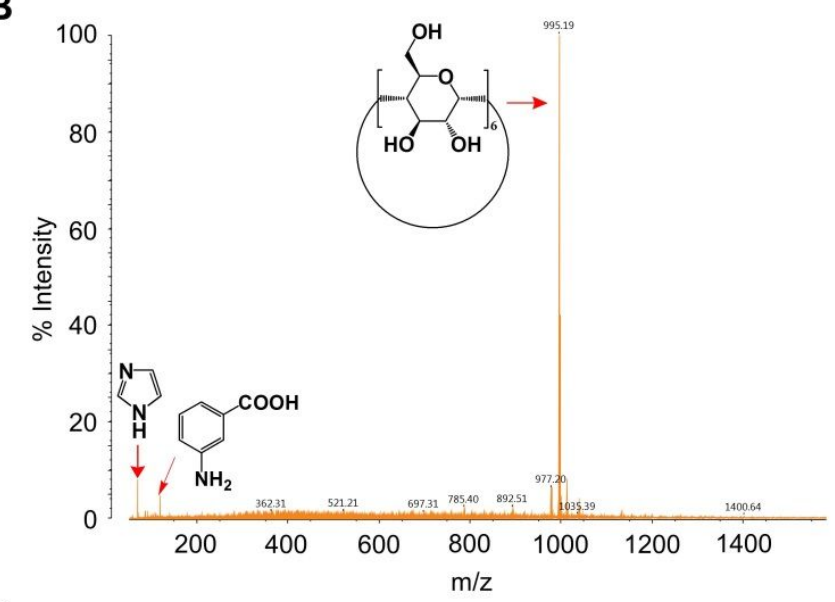

D

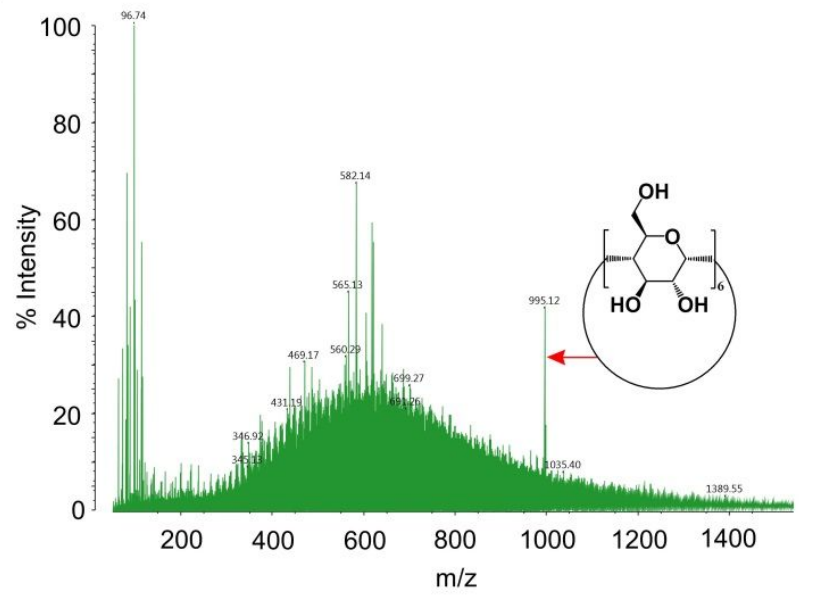

Figure S6. Hydrolysis of LaCD NP in biological samples. (A-D) ${ }^{1} \mathrm{H}$ NMR spectra (A,C) and MALDI-TOF mass spectra $(\mathrm{B}, \mathrm{D})$ of hydrolyzed products of LaCD NP after incubation with PMA-stimulated neutrophils $(\mathrm{A}, \mathrm{B})$ or the liver homogenates $(\mathrm{C}, \mathrm{D})$ for 7 days. In both cases, hydrolyzed water-soluble products in the supernatant were lyophilized for ${ }^{1} \mathrm{H}$ NMR measurement in $\mathrm{D}_{2} \mathrm{O}$ or MALDI-TOF mass spectrometry. 
A

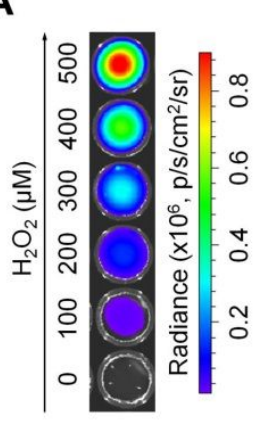

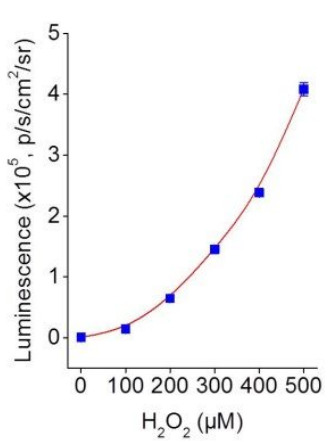

B

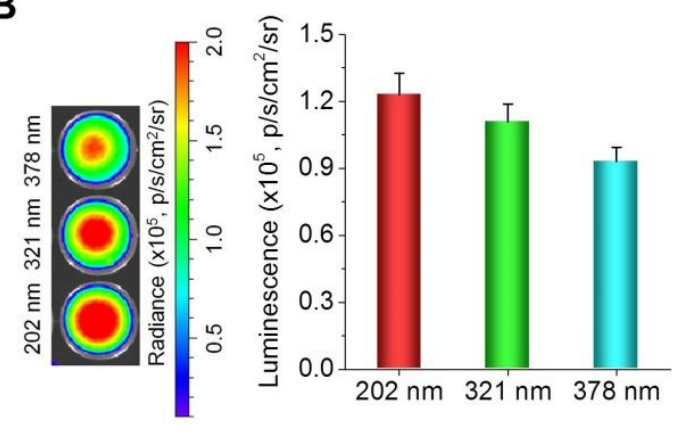

C

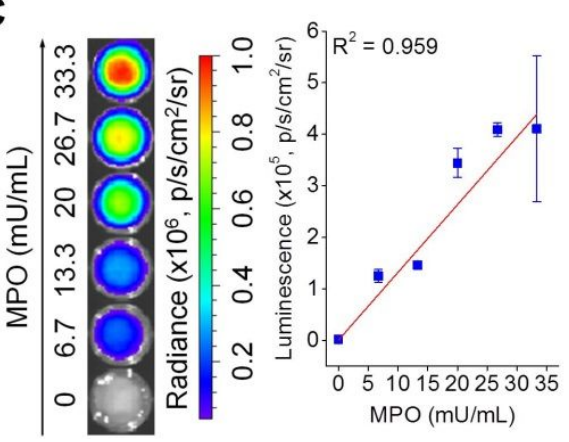

D

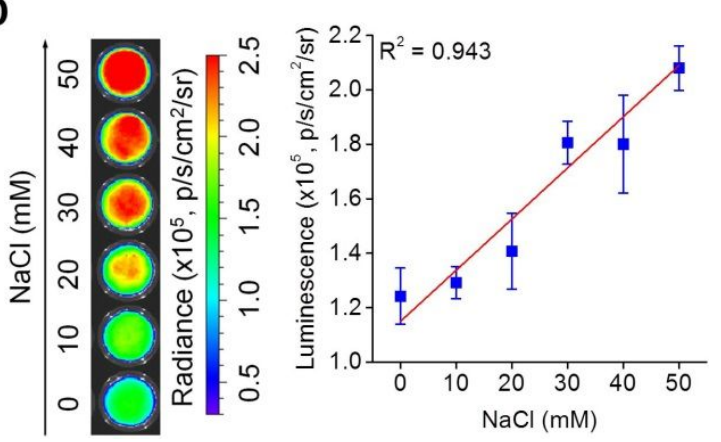

Figure S7. Luminescence properties of LaCD NPs. (A) Luminescent intensities of LaCD NP at high levels of $\mathrm{H}_{2} \mathrm{O}_{2}$. (B) The effect of particle size on luminescence of LaCD NPs. (C,D) The effects of MPO (C) or $\mathrm{Cl}^{-}$(D) levels on luminescence of LaCD NP. In all cases, the left panels show typical luminescent images, while the right panels illustrate quantitative results. Data are means $\pm \operatorname{SD}(n=3)$. 
A

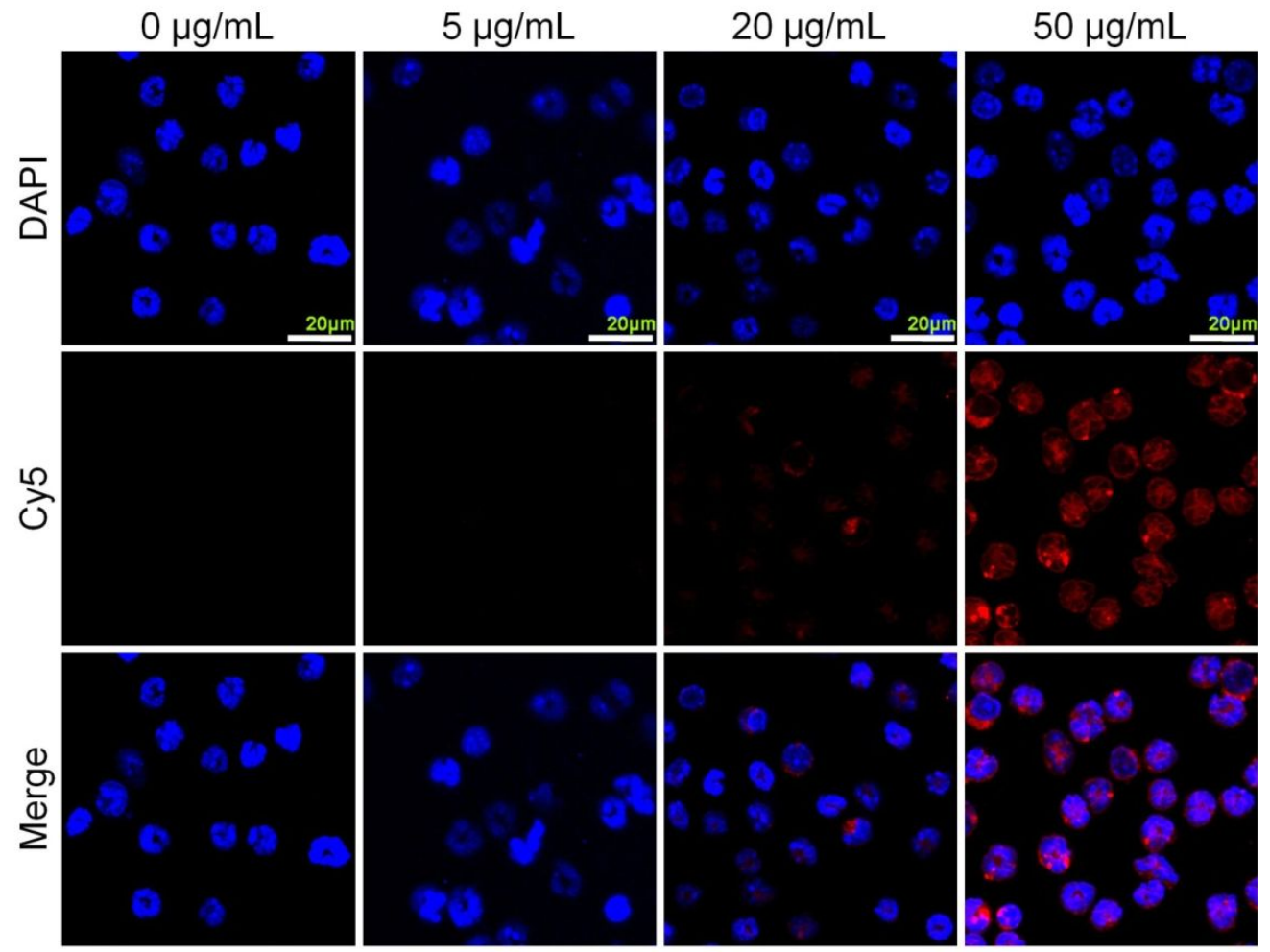

B

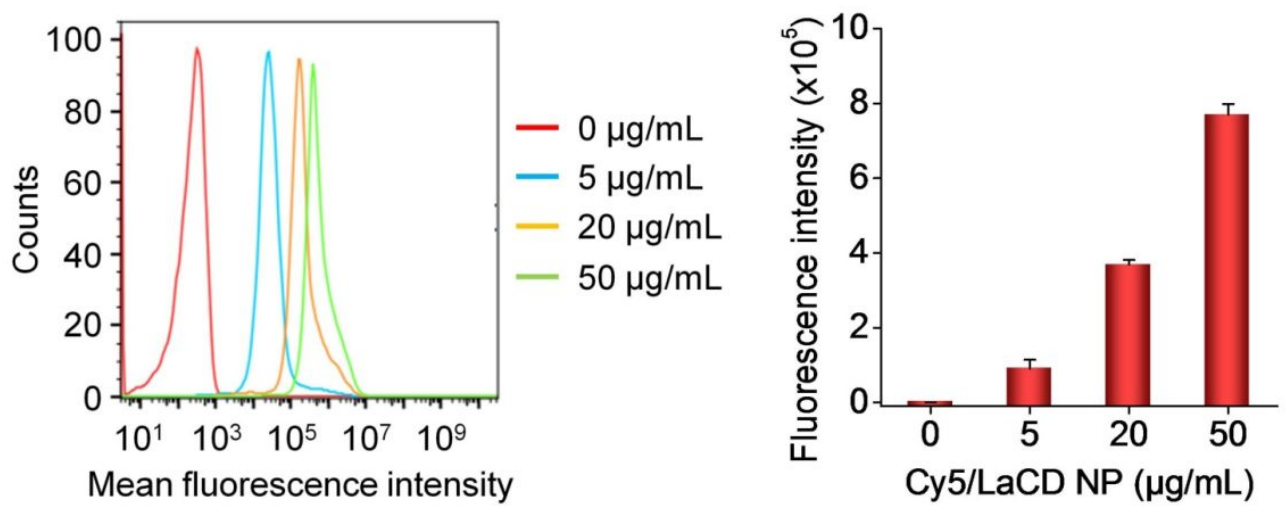

Figure S8. Dose-dependent cellular uptake of LaCD NP in mouse neutrophils. (A) Confocal microscopic images showing cellular internalization of Cy5-labeled LaCD NP at different doses after $1 \mathrm{~h}$ of incubation. (B) Flow cytometry quantification of internalized $\mathrm{Cy} 5 / \mathrm{LaCD} \mathrm{NP}$ in neutrophils. Data are presented as means $\pm \mathrm{SD}$ $(\mathrm{n}=3)$. 
A

B
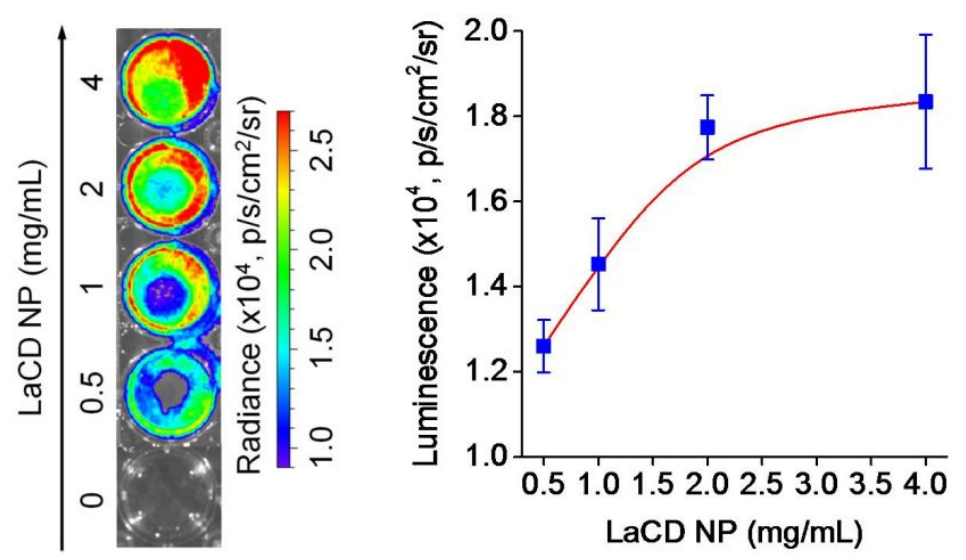

Figure S9. Luminescence imaging of activated neutrophils with LaCD NP. (A) Representative luminescent signals of stimulated neutrophils incubated with different doses of LaCD NP. (B) The quantified data. Data are presented as means $\pm \operatorname{SD}(n=3)$. 
A
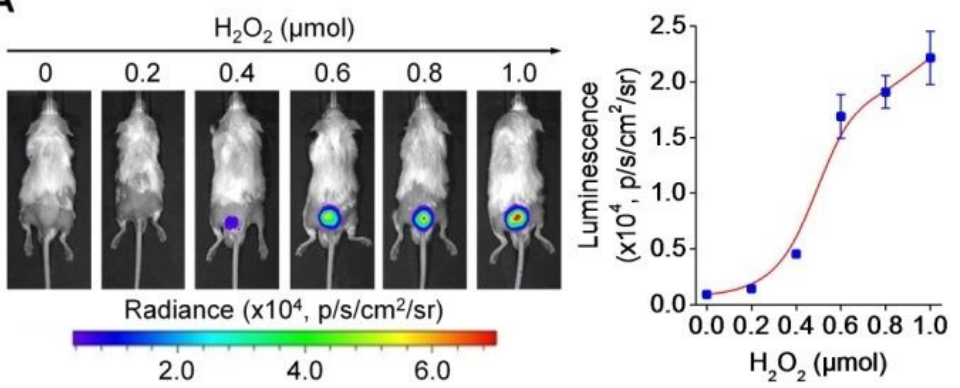

B
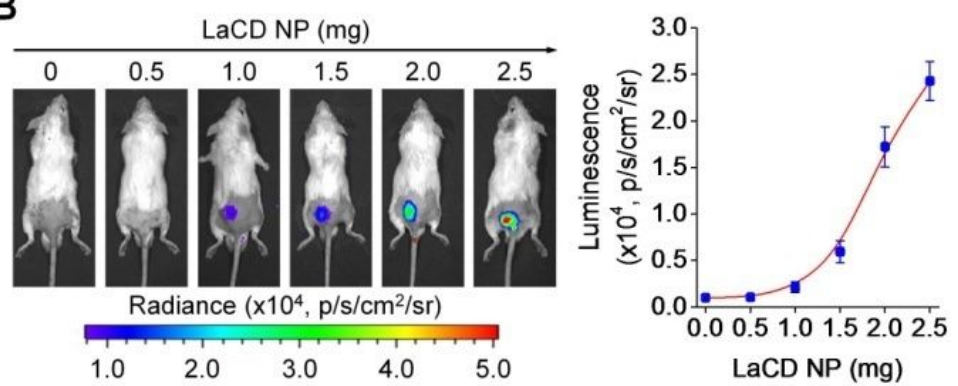

Figure S10. Luminescence imaging of ROS in mice with LaCD NP. (A) In vivo luminescent signals in mice subcutaneously injected with different doses of $\mathrm{H}_{2} \mathrm{O}_{2}$ and $2 \mathrm{mg} \mathrm{LaCD} \mathrm{NP}$ in each mouse. (B) Luminescent intensities of mice subcutaneously administered with different doses of LaCD NP and $1 \mu$ mol $\mathrm{H}_{2} \mathrm{O}_{2}$. In both cases, the left panels show representative in vivo images, while the right panels illustrate quantitative data. Data are presented as means $\pm \mathrm{SD}(\mathrm{n}=3)$. 
A

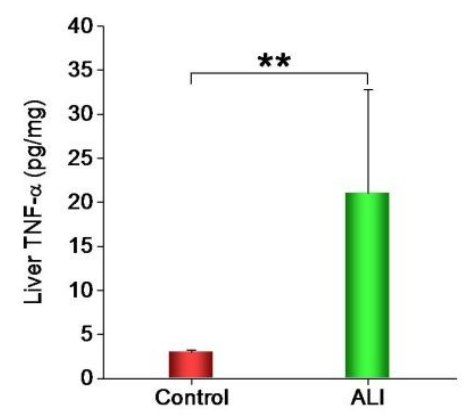

C

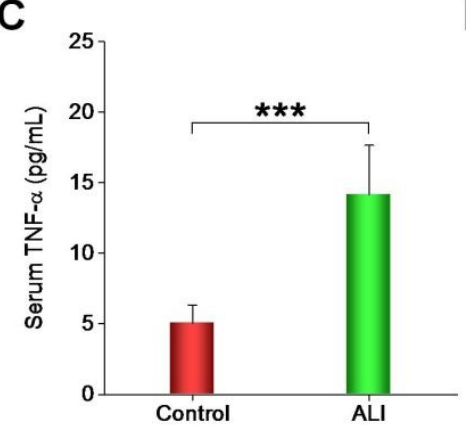

B

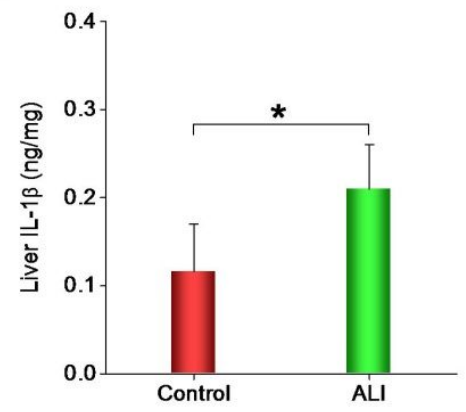

D

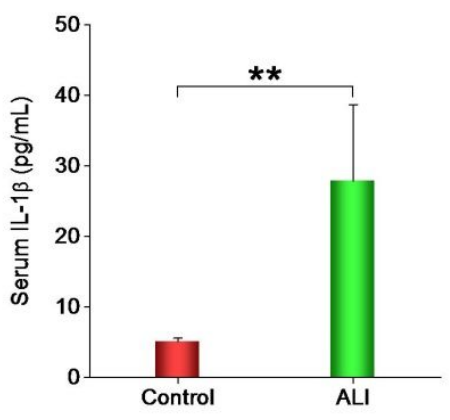

Figure S11. Expression levels of typical inflammatory cytokines in ALI mice. (A,B) Levels of TNF- $\alpha$ (A) and IL-1 $\beta$ (B) in hepatic tissues of ALI mice. (C,D) Serum levels of TNF- $\alpha$ (C) and IL-1 $\beta$ (D). Data are presented as means $\pm \mathrm{SD}(\mathrm{n}=6) .{ }^{*} P<0.05,{ }^{* *} P<0.01,{ }^{* * *} P<0.001$. 
A
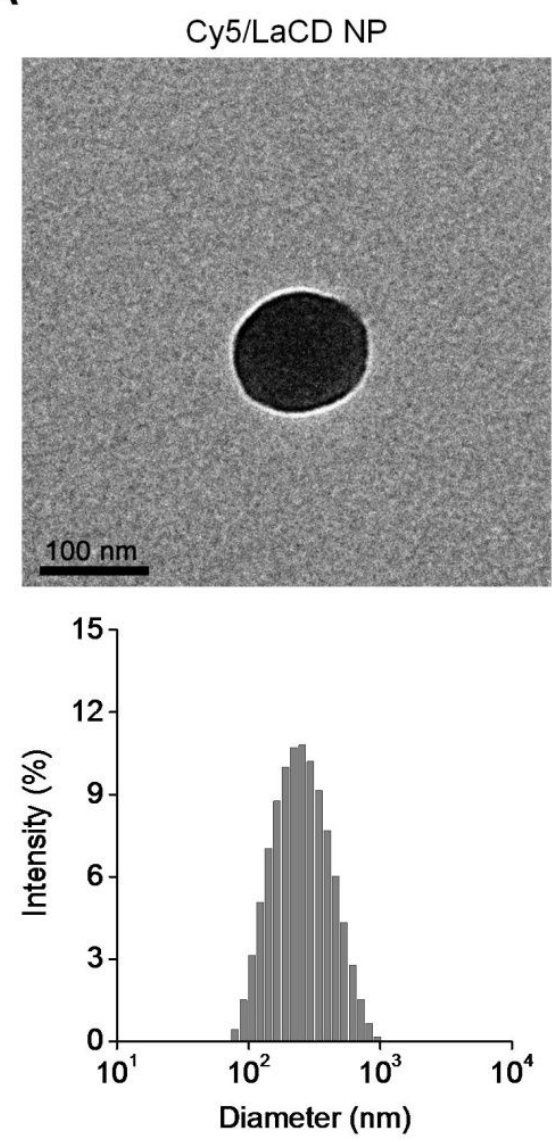

B
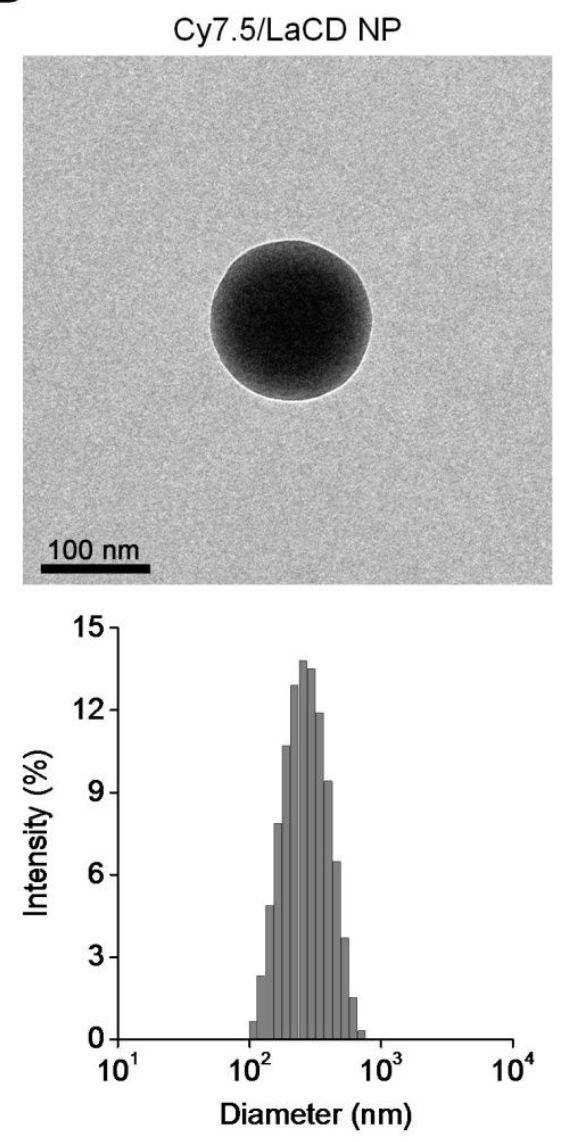

Figure S12. Characterization of fluorescent-labeled LaCD NPs. (A,B) TEM images (upper) and size distribution (lower) of Cy5-labeled $\mathrm{LaCD} \mathrm{NP}$ (A) or Cy7.5-labeled $\mathrm{LaCD} \mathrm{NP}$ (B) produced by nanoprecipitation. 
A

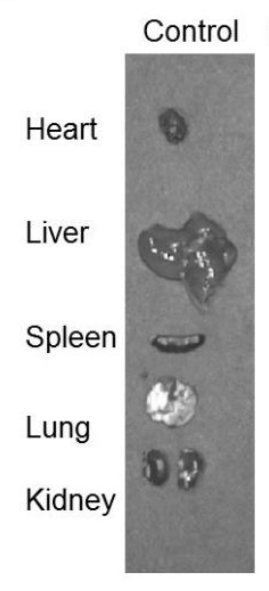

Cy7.5/LaCD NP

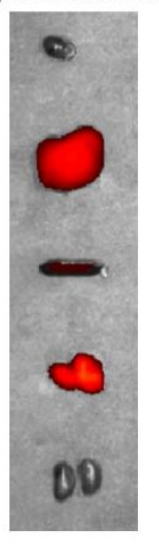

B

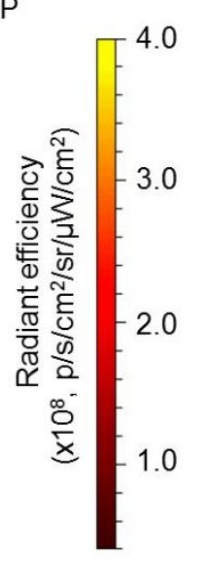

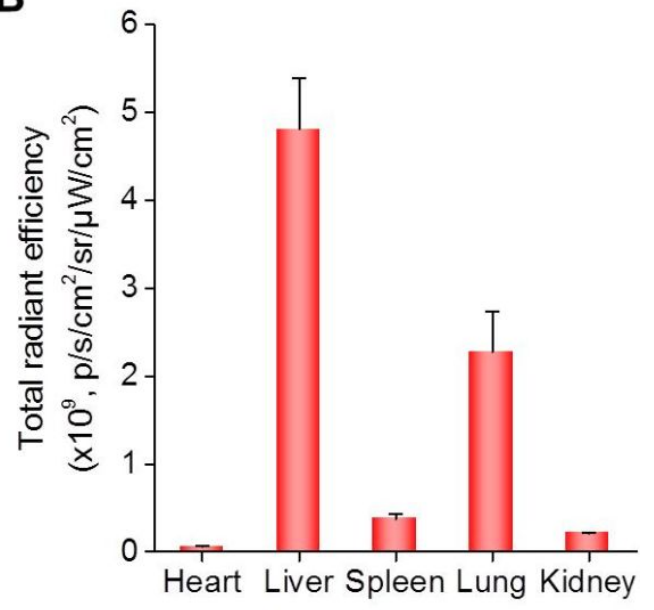

Figure S13. Accumulation of Cy7.5/LaCD NP in typical major organs. (A,B) Ex vivo images (A) and quantitative data (B) showing the total fluorescence intensities of Cy7.5/LaCD NP in heart, liver, spleen, lung, and kidney of ALI mice after i.v. administration for 15 min. Data are presented as means $\pm \mathrm{SD}(\mathrm{n}=3)$. 


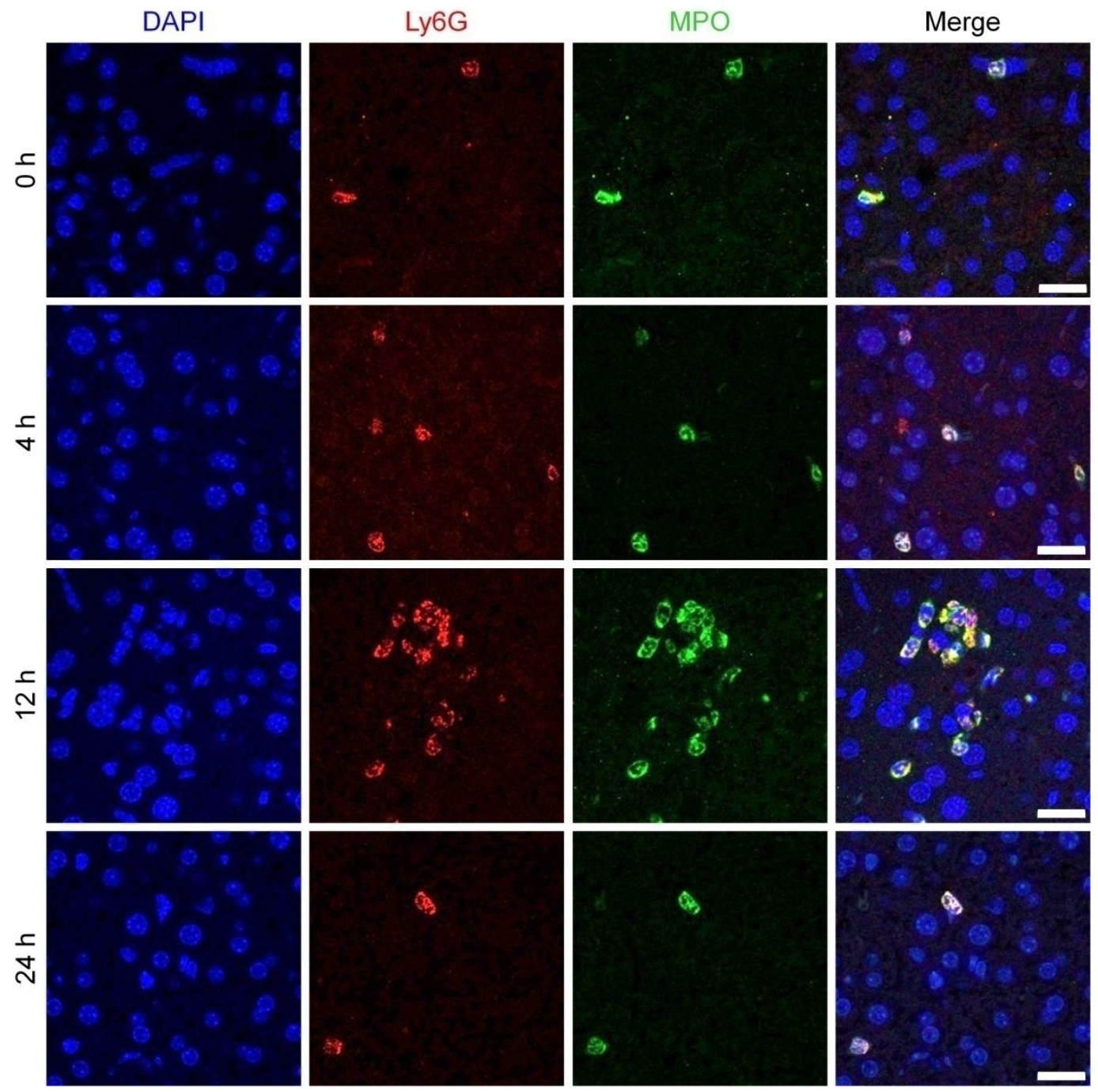

Figure S14. Immunofluorescence analysis of neutrophils and MPO in liver tissues from ALI mice. The hepatic tissues were isolated from mice at different time points after oral administration of 50\% ethanol. The liver sections were stained with Cy3-Ly6G antibody (red) and FITC-MPO antibody (green) for detection of neutrophils and MPO, respectively. Nuclei were counterstained with DAPI. Scale bars, $20 \mu \mathrm{m}$. 

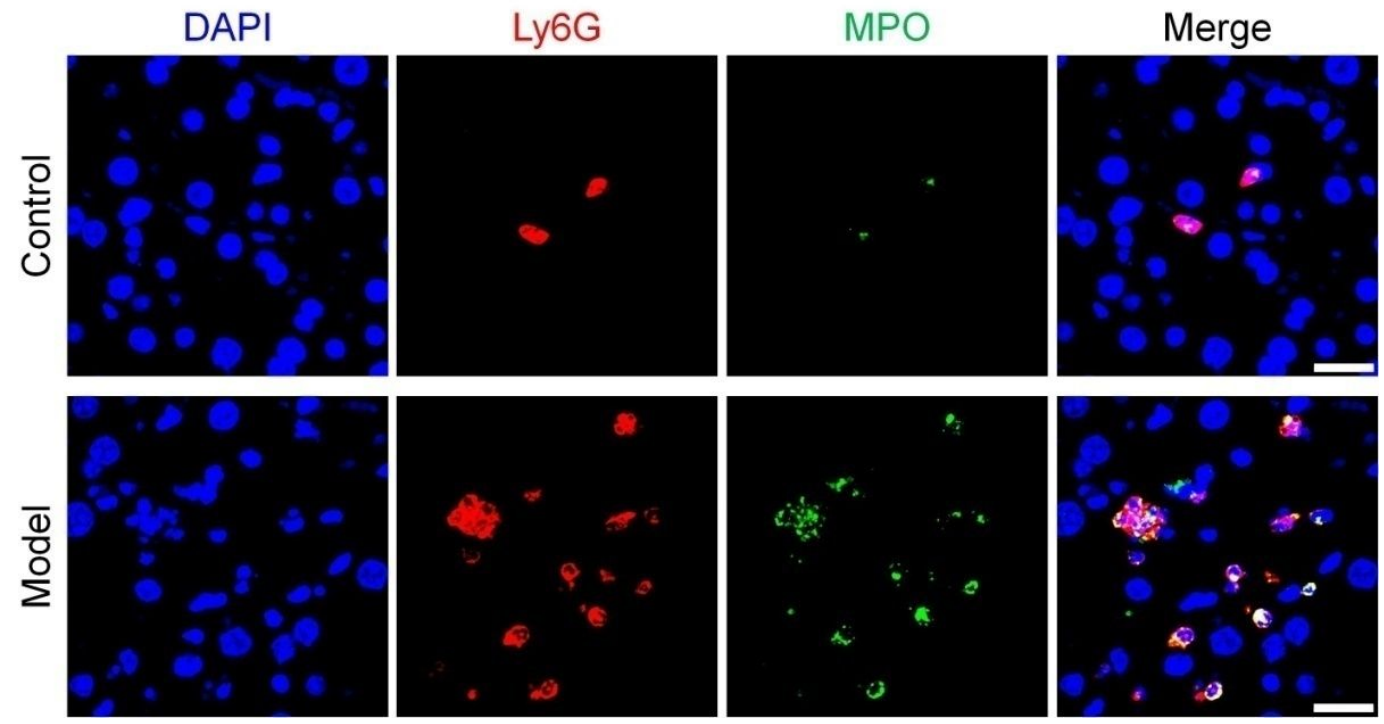

Figure S15. Immunofluorescence analysis of neutrophils and MPO in liver tissues of mice with ALF induced by $\mathrm{CCl}_{4}$. The hepatic tissues were isolated from mice at $24 \mathrm{~h}$ after i.p. administration of $\mathrm{CCl}_{4}$. The liver sections were stained with Cy3-Ly6G antibody (red) and FITC-MPO antibody (green) for detection of neutrophils and MPO, respectively. Nuclei were counterstained with DAPI. Scale bars, $20 \mu \mathrm{m}$. 

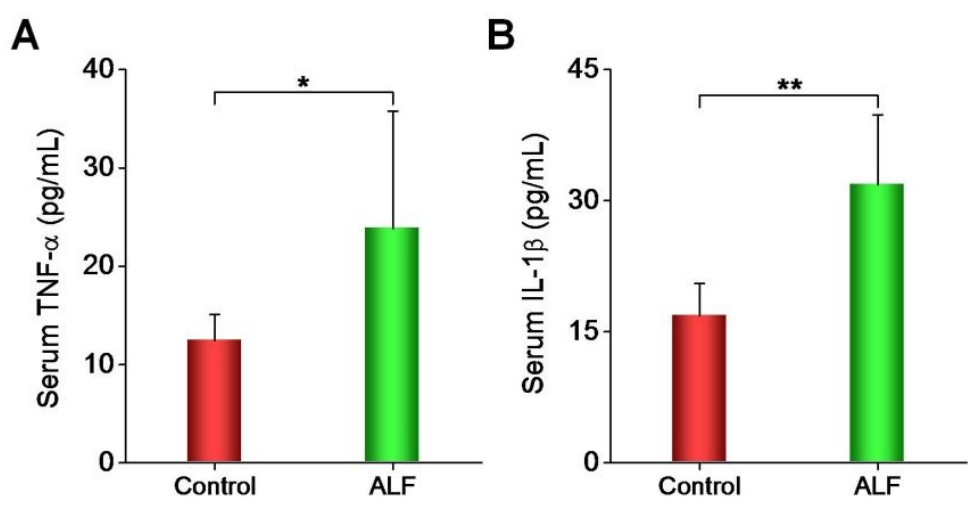

Figure S16. Expression levels of typical inflammatory cytokines in serum from ALF mice. (A,B) Serum levels of TNF- $\alpha(\mathrm{A})$ and IL-1 $\beta(\mathrm{B})$. Data are presented as means $\pm \mathrm{SD}(\mathrm{n}=6) .{ }^{*} P<0.05,{ }^{* *} P<0.01$. 
A

B
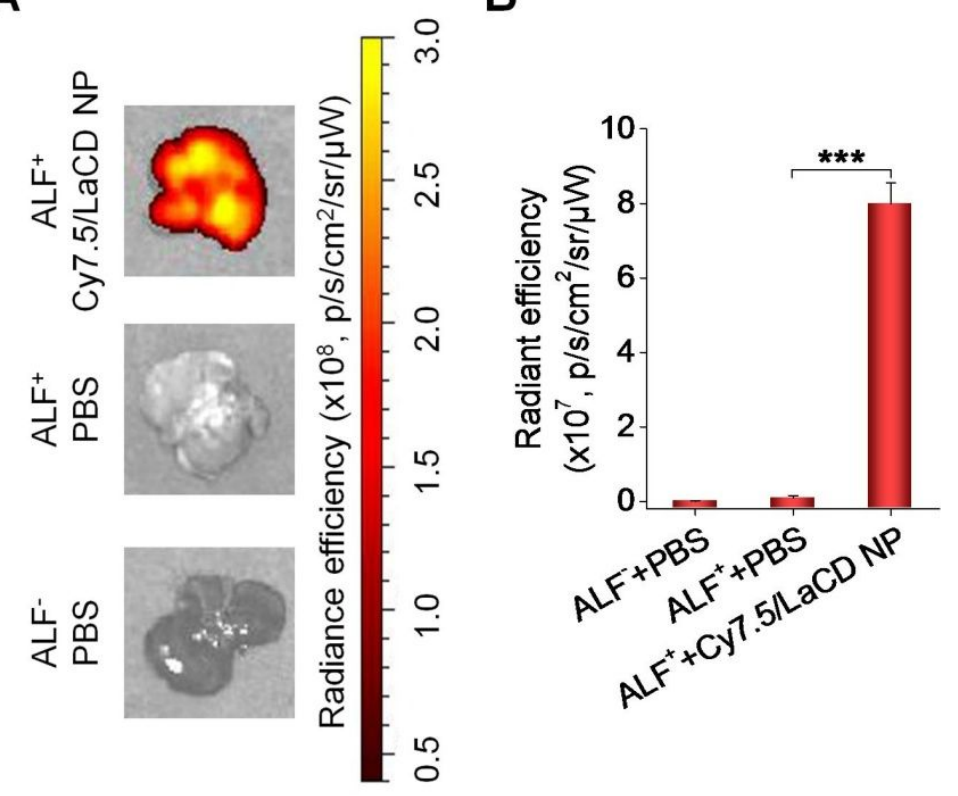

Figure S17. Targeting liver injury in ALF mice. (A,B) Ex vivo fluorescence images (A) and quantitative data (B) indicate the liver accumulation of Cy7.5-labeled LaCD NP at $10 \mathrm{~min}$ after i.v. injection in mice with $\mathrm{CCl}_{4}$-induced ALF. Data are presented as means $\pm \mathrm{SD}(\mathrm{n}=4) .{ }^{* * *} P<0.001$. 


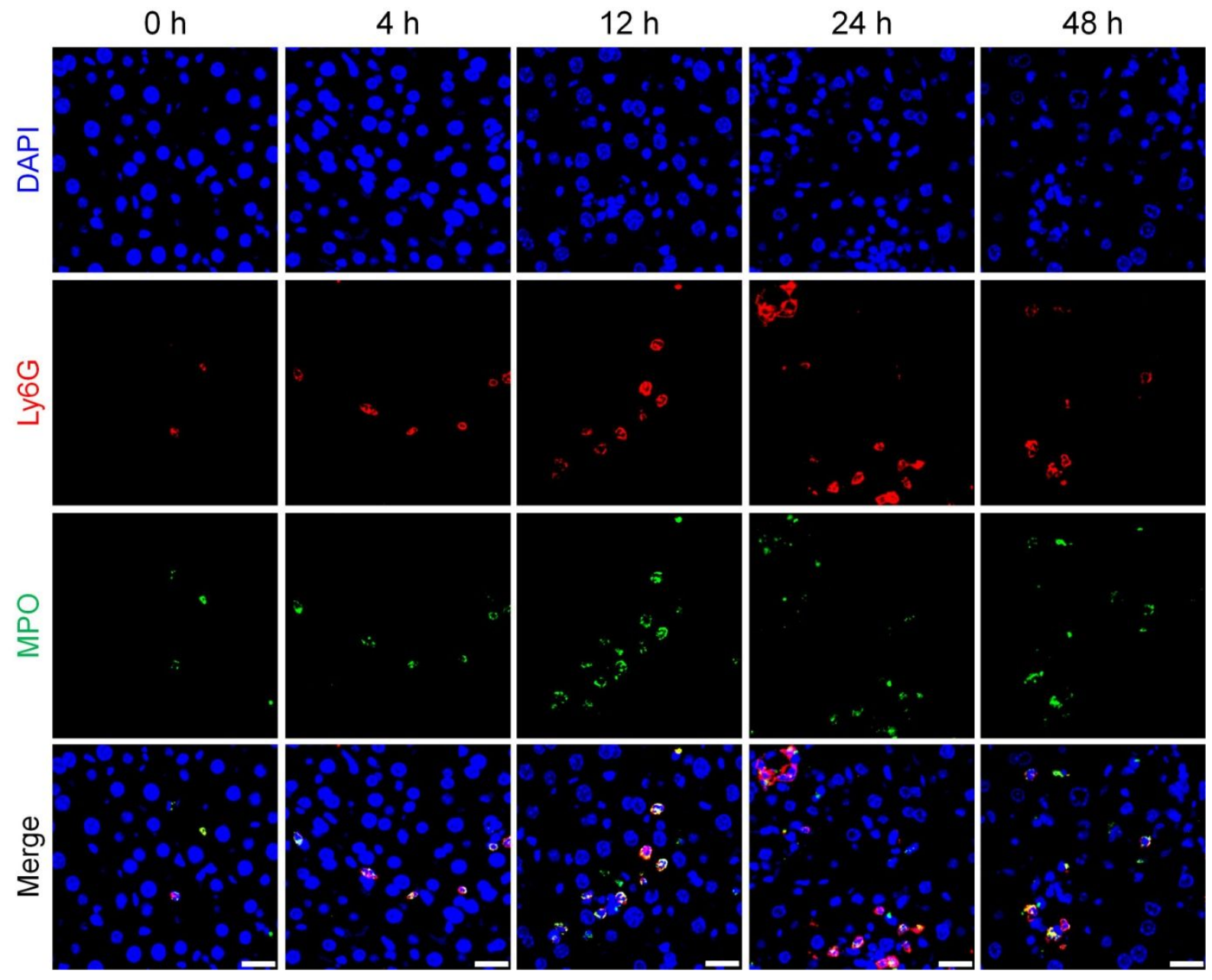

Figure S18. Immunofluorescence analysis of neutrophils and MPO in liver tissues from ALF mice at different stages. The hepatic tissues were isolated from mice at different time points after stimulation with $\mathrm{CCl}_{4}$ administered by i.p. injection. The liver sections were stained with Cy3-labeled anti-Ly6G antibody (red) and FITC-labeled anti-MPO antibody (green) for detection of neutrophils and MPO, respectively. Nuclei were counterstained with DAPI. Scale bars, $20 \mu \mathrm{m}$. 
A

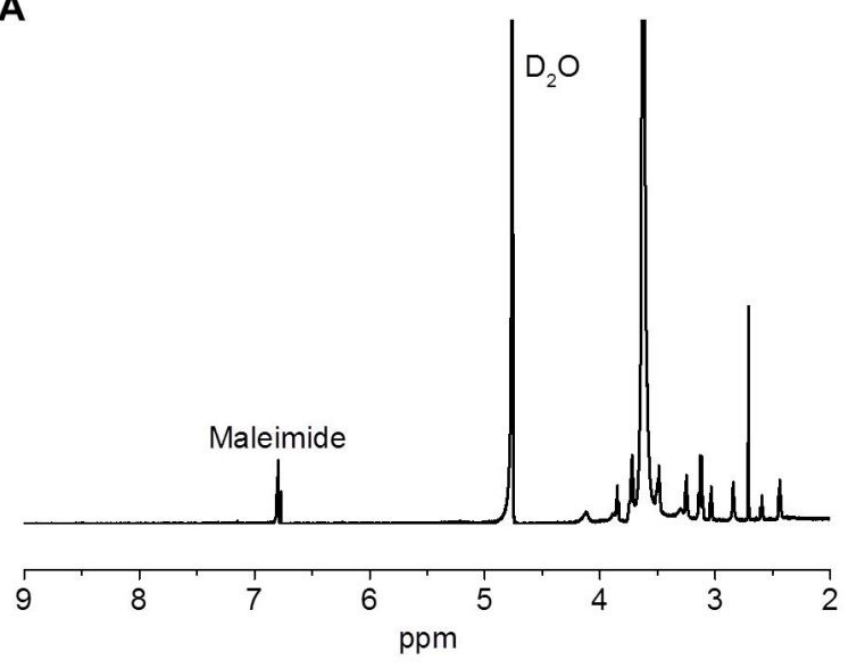

B
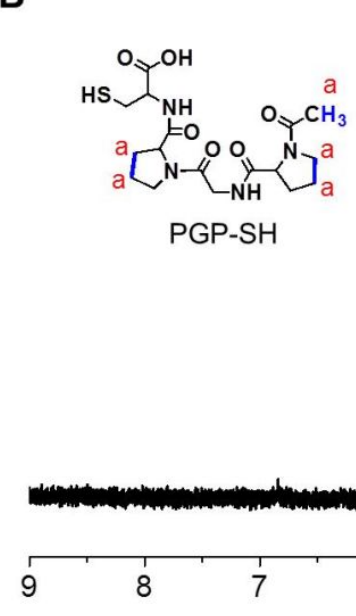

Figure S19. ${ }^{1} \mathrm{H}$ NMR spectroscopy characterization of PGP-conjugated DSPE-PEG. (A,B) ${ }^{1} \mathrm{H}$ NMR spectra of DSPE-PEG-Maleimide (A) and PGP-conjugated DSPE-PEG (B) in $\mathrm{D}_{2} \mathrm{O}$. After conjugation with PGP-SH, the proton signals corresponding to maleimide in DPSE-PEG-Maleimide disappeared. 

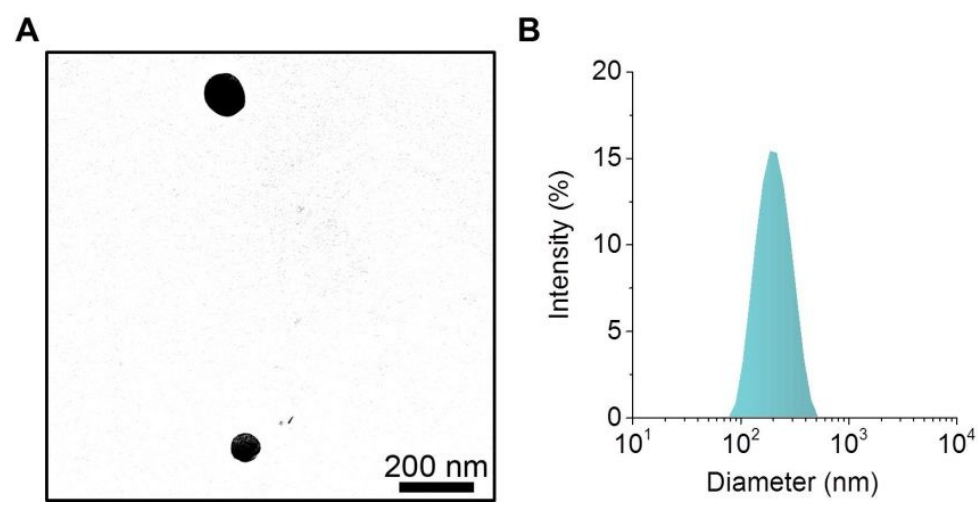

Figure S20. Characterization of a control nanoprobe of PEG-coated LaCD NP (LaCD-PEG NP). (A,B) TEM image (A) and size distribution (B) of LaCD-PEG NP. The mean diameter of LaCD-PEG NP was approximately $210 \mathrm{~nm}$. 
A
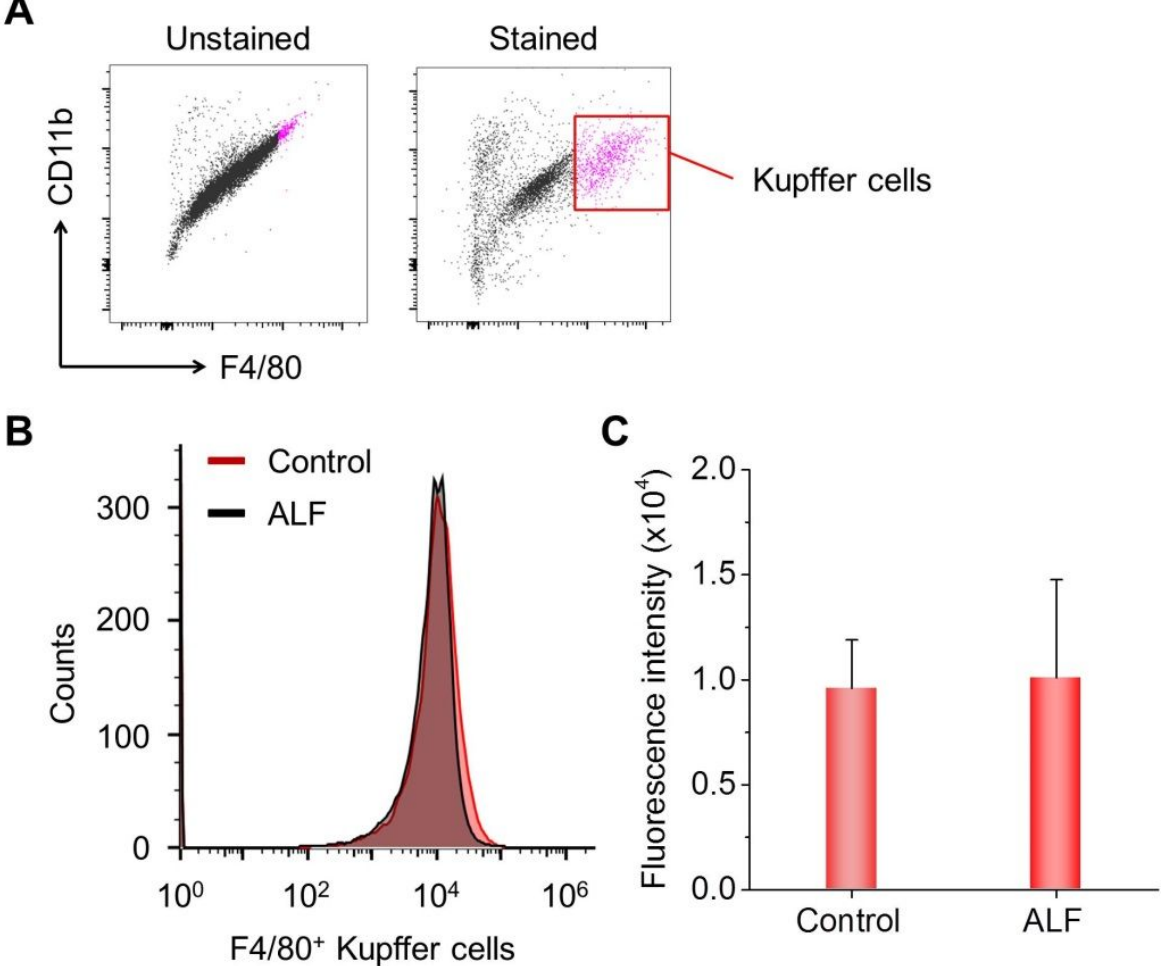

Figure S21. Analysis of Kupffer cells in ALF mice. (A) Identification of Kupffer cells in the liver isolated from C57BL/6 mice by flow cytometry. (B,C) Representative flow cytometric profiles (B) and quantified fluorescence intensities (C) of FITC-labeled Kupffer cells isolated from livers of either healthy mice (Control) or mice with $\mathrm{CCl}_{4}$-induced ALF. Data are presented as means $\pm \mathrm{SD}(\mathrm{n}=5)$. 
A

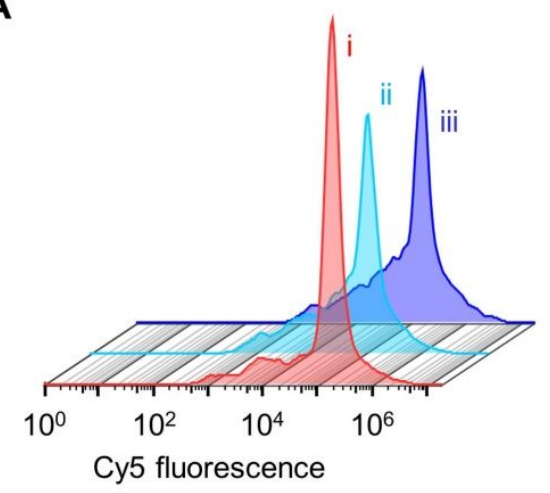

B

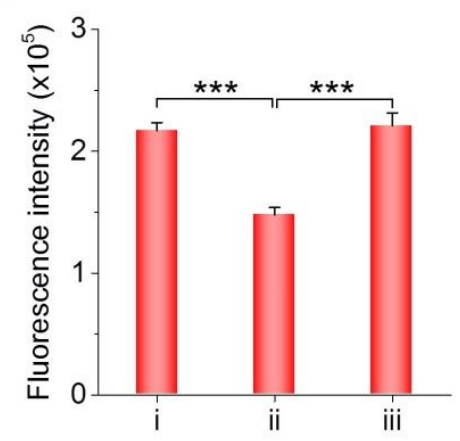

C

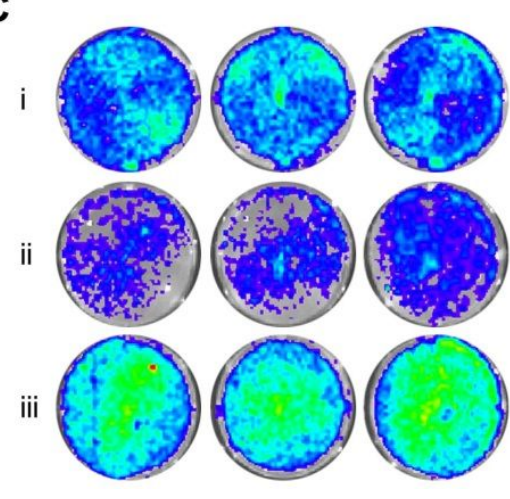

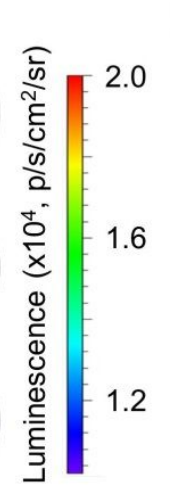

D

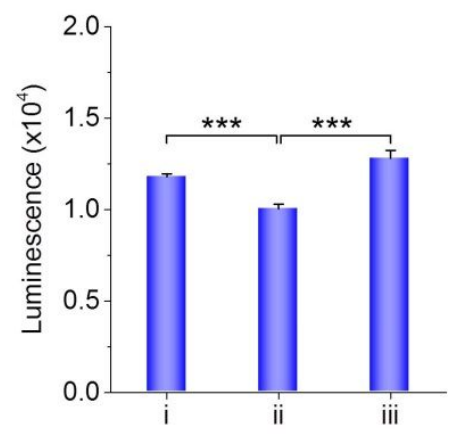

Figure 22. The effects of macrophages on cellular uptake and luminescence of LaCD-PEG NP and LaCD-PEG-PGP NP in neutrophils. (A,B) Representative flow cytometric profiles (A) and quantified fluorescence intensities (B) showing cellular internalization of Cy5/LaCD-PEG NP or Cy5/LaCD-PEG-PGP NP in neutrophils after $1 \mathrm{~h}$ of incubation with or without co-culture with macrophages. The dose of Cy5/LaCD-PEG NP or Cy5/LaCD-PEG-PGP NP was $50 \mu \mathrm{g} / \mathrm{mL}$. (C,D) In vitro luminescence images (C) and quantified luminescence intensities (D) showing the influence of macrophages on luminescence profiles of LaCD-PEG NP or LaCD-PEG-PGP NP in neutrophils. In both cases, the count ratio of neutrophils to macrophages was 4:3. Data are presented as means $\pm \mathrm{SD}(\mathrm{B}, \mathrm{n}=4 ; \mathrm{D}, \mathrm{n}=3)$. $* * * P<0.001$ 

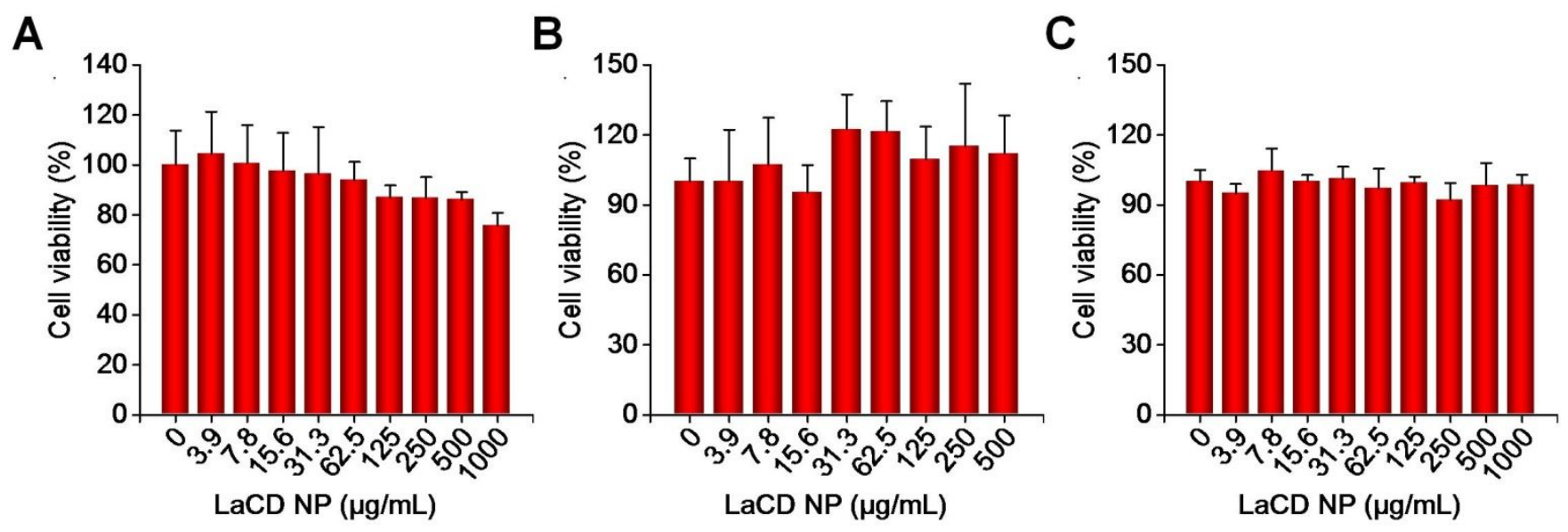

Figure S23. In vitro cytotoxicity evaluations of LaCD NP in different cell lines. (A-C) Cell viability of RAW264.7 murine macrophages (A), human umbilical vein endothelial cells (HUVECs) (B), and HepG2 human liver cancer cells (C) after incubation with different doses of LaCD NP for $12 \mathrm{~h}$. Data are presented as means $\pm \operatorname{SD}(n=6)$. 

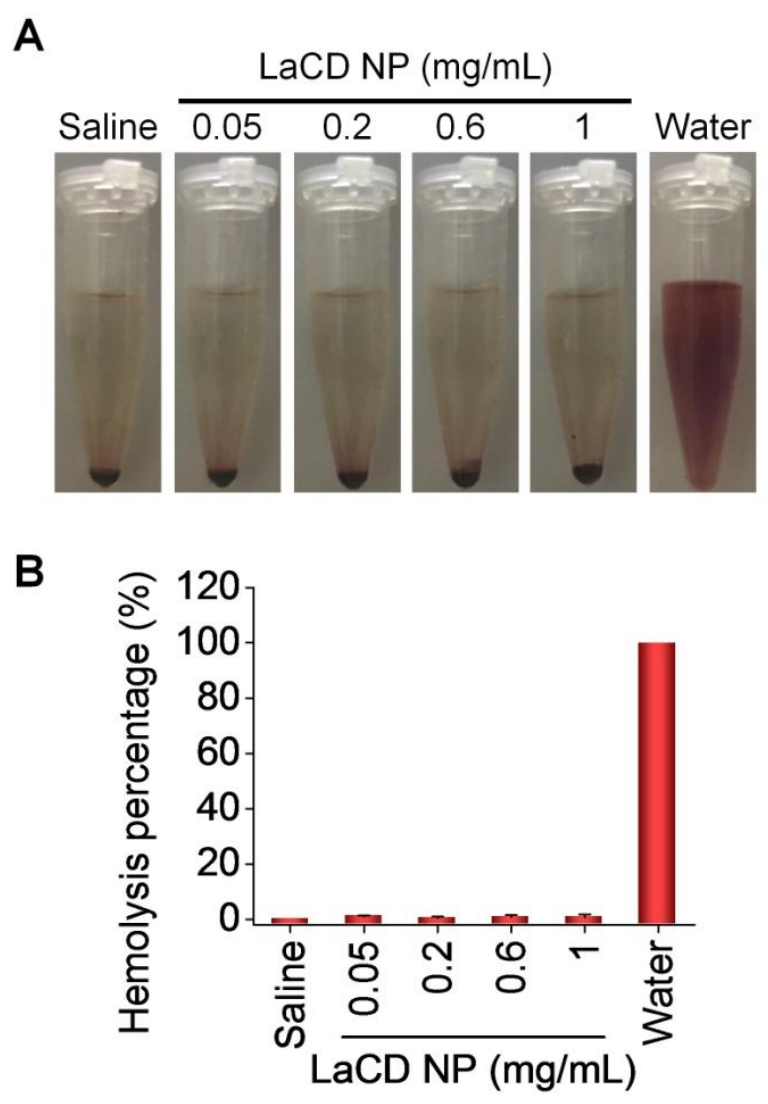

Figure S24. Hemolysis tests of LaCD NP. (A) Digital photos showing aqueous suspensions of erythrocytes isolated from rat blood treated with saline (the control group), LaCD NP at various concentrations, or pure water at $37^{\circ} \mathrm{C}$ for $2 \mathrm{~h}$, and then centrifuged at $200 \mathrm{~g}$ for $5 \mathrm{~min}$ to give a clear presentation. (B) The degree of hemolysis was calculated by quantifying the optical density of hemoglobin at $570 \mathrm{~nm}$. Data are presented as means $\pm \operatorname{SD}(n=4)$. 
A
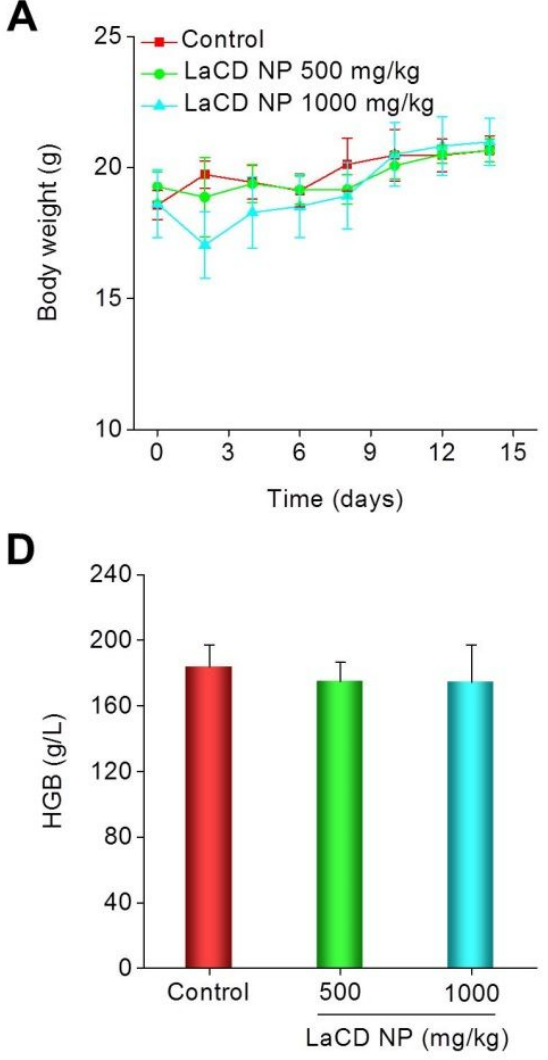

B

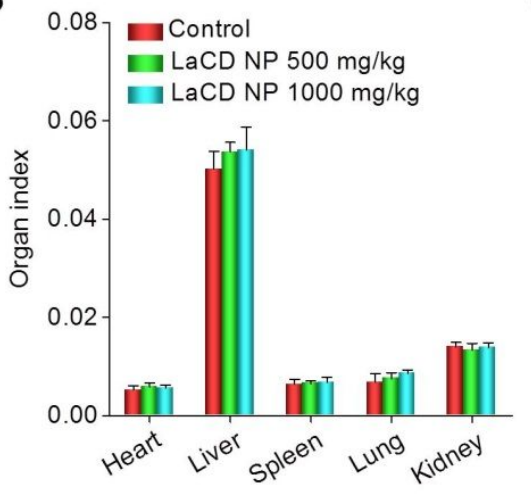

$\mathbf{E}$

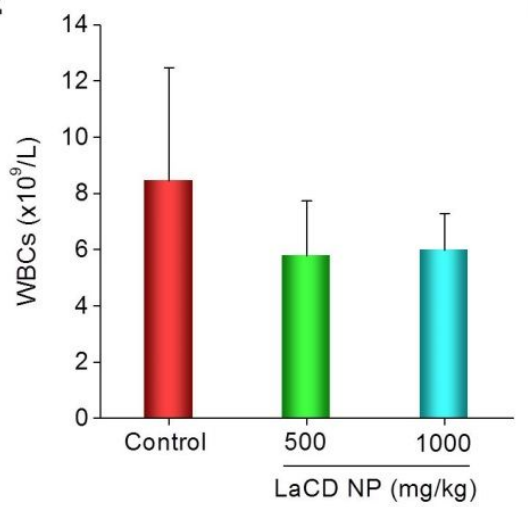

C

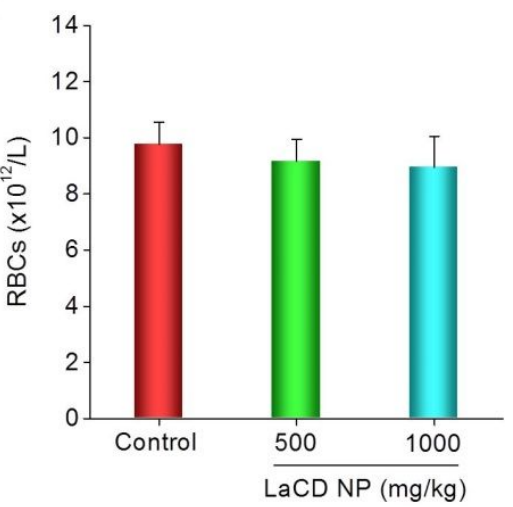

$\mathbf{F}$

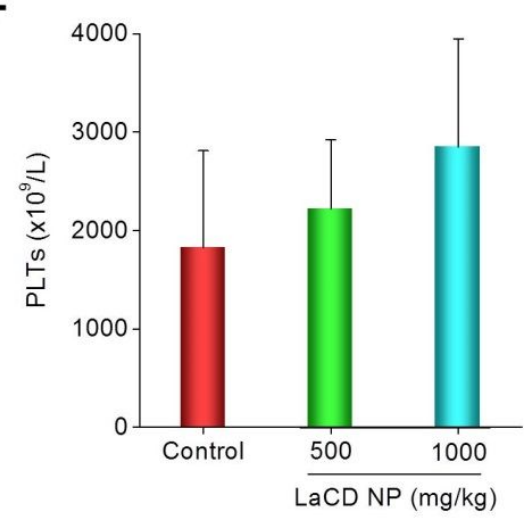

Figure S25. Acute toxicity evaluation of LaCD NP in mice. (A) Changes in body weight of BALB/c mice after a single i.v. injection of different formulations. The control group was treated with saline, while mice in the other two groups were administered with LaCD NP at 500 or $1000 \mathrm{mg} / \mathrm{kg}$. (B) The organ index of major organs excised from mice at day 15 after different treatments. (C-F) Typical hematological parameters including RBCs (C), HGB (D), WBCs (E), and PLTs (F). RBCs, red blood cells; HGB, hemoglobin; WBCs, white blood cells; PLTs, platelets. Data are presented as means \pm SD $(n=6)$. 
A

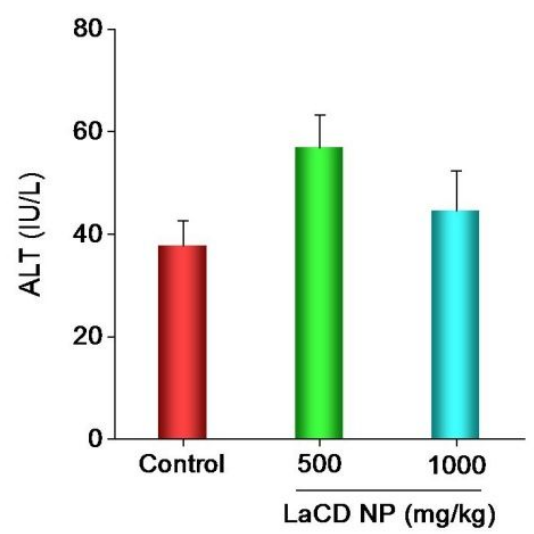

C

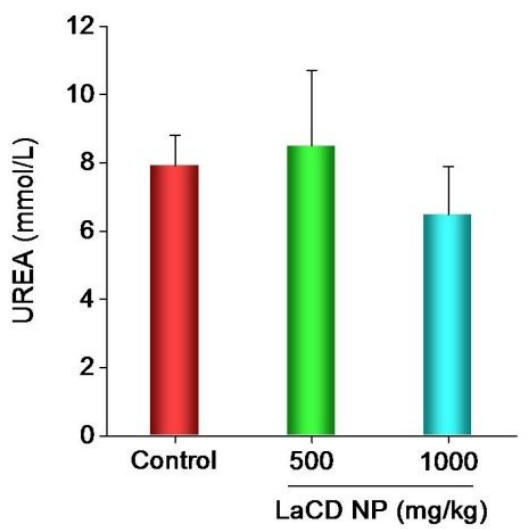

B

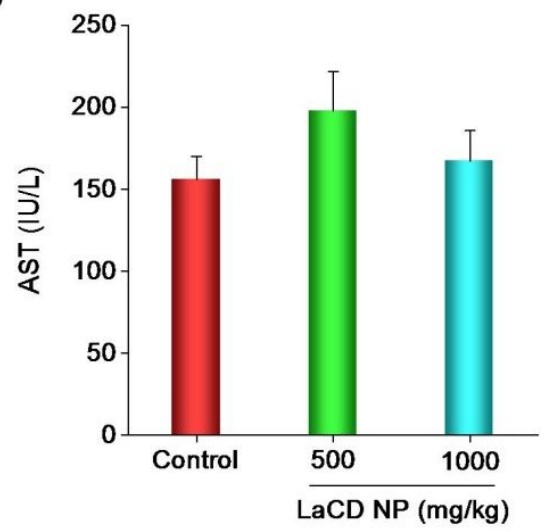

D

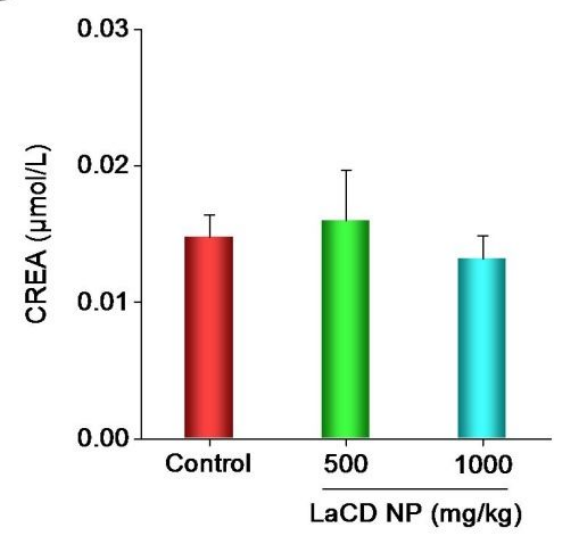

Figure S26. Changes in the levels of typical biomarkers related to hepatic and kidney functions. (A-D) The serum levels of ALT (A), AST (B), UREA (C), and CREA (D) at day 15 after different treatments. ALT, alanine aminotransferase; AST, aspartate aminotransferase; UREA, blood urea; CREA, creatinine. Data are presented as means $\pm \operatorname{SD}(n=6)$. 


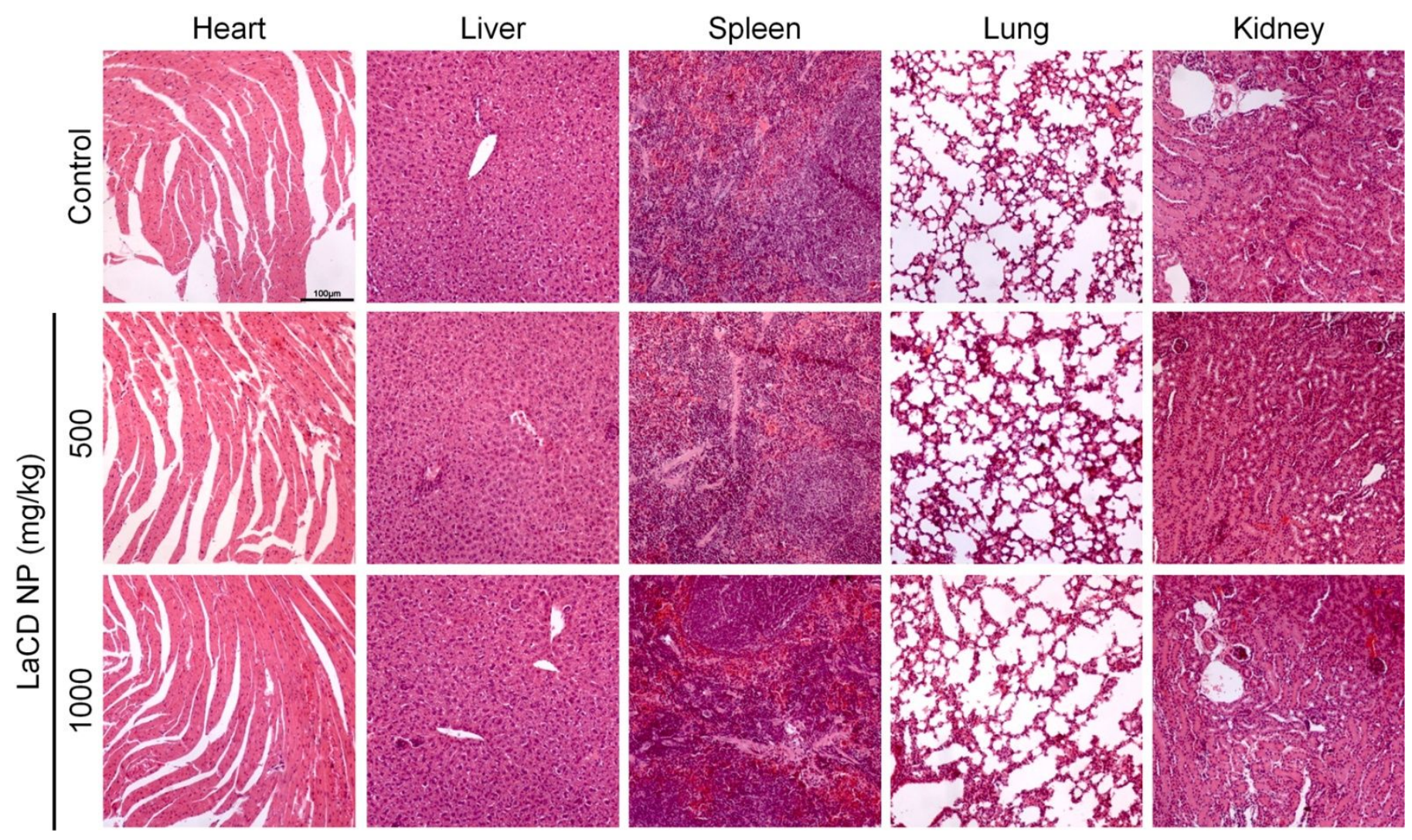

Figure S27. H\&E stained histological sections of major organs. At day 15 after treatment with a single dose of $\mathrm{LaCD} \mathrm{NP}$ at 500 or $1000 \mathrm{mg} / \mathrm{kg}, \mathrm{BALB} / \mathrm{c}$ mice were euthanized and typical organs such as heart, liver, spleen, lung, and kidney were isolated for analysis. 